\title{
PROPAGATION OF SINGULARITIES FOR NONSTRICTLY HYPERBOLIC SEMILINEAR SYSTEMS IN ONE SPACE DIMENSION ${ }^{1}$
}

BY

\author{
LUCIO MICHELI
}

\begin{abstract}
We consider the creation and propagation of singularities in the solutions of semilinear nonstrictly hyperbolic systems in one space dimension when the initial data has jump discontinuities. We show that singularities travelling along characteristics can branch at points of degeneracy of the vector fields on all other forward characteristics.

We prove a lower bound for the strength of these new singularities, and we give an example showing that our result cannot be improved in general.
\end{abstract}

1. Introduction. In the last few years the problem of the creation and propagation of singularities for strictly hyperbolic semilinear systems has been studied in great detail (see $[1,2,3])$, but a recent example [4] has shown that new phenomena can occur when characteristics of variable multiplicity are present.

In this paper we study the Cauchy problem for a nonstrictly hyperbolic semilinear system in one space dimension when the initial data $\mathbf{u}^{0}$ is in $C^{\infty}$ except for finitely many jump discontinuities in $\mathbf{u}^{0}$ or in its derivatives. We restrict ourselves to diagonal systems in which exactly two families of characteristic curves are tangent along a smooth noncharacteristic curve while all others are transversal. We also assume that the order of contact is constantly equal to $p-1, p$ even, and the coefficients of the system are smooth.

A locally bounded solution $\mathbf{u}$ of our system is uniquely determined by its initial data $\mathbf{u}^{0}$, and it is natural to consider the problem of describing the strength and the location of the singularities of $\mathbf{u}$. We say that $\mathbf{u}$ has order $m$ across a curve if all of its first $m$ partial derivatives are continuous across the curve, but the same is not true for $m+1$. Jump discontinuities have order -1 . The typical situation that we are going to study is sketched in Figure 1.1.

We consider a characteristic $C_{1}$ carrying a singularity of order $n_{1}$, and we assume that at point $\mathbf{P}$ there is a characteristic $C_{2}$ tangent to $C_{1}$ with an order of contact equal to $p-1$. We assume also that the other characteristics through $\mathbf{P}$ are transversal and there are no other incoming singularities.

First of all there is a linear phenomenon: a singularity travelling along a characteristic can branch at a point of degeneracy onto the tangent characteristic,

Received by the editors January 3, 1984.

1980 Mathematics Subject Classification. Primary 35L60.

${ }^{1}$ This research was partially supported by a C.N.R. grant. 


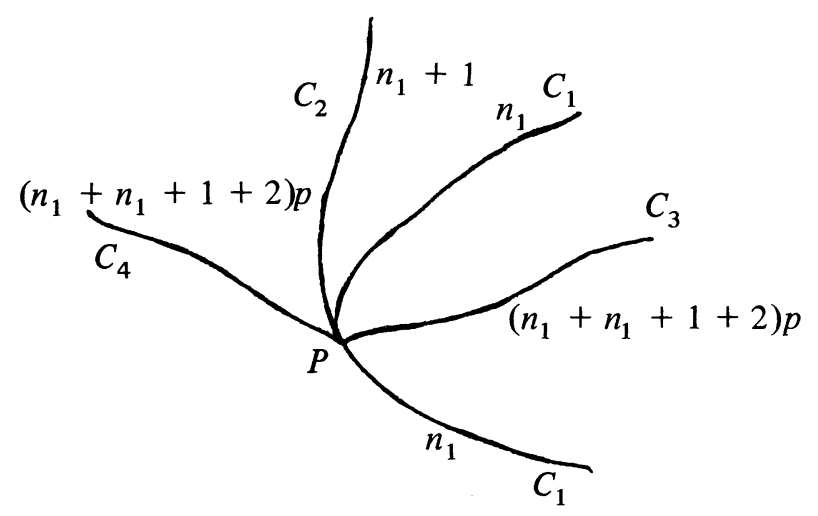

FIGURE 1.1

and it is easy to show that, in the case of Figure 1.1, the solution is of order $n_{1}+1$ across $C_{2}$.

Then there is a nonlinear phenomenon: the point of degeneracy of a characteristic bearing singularity is a source of new singularities travelling on all forward characteristics from that point. It is hard to determine the strength of these new singularities, as it depends not only on the strength of the incoming singularity but also on the order of contact of the tangent characteristics. Rauch and Reed conjectured in [4] that the right answer was given by the formula

$$
n_{1}+n_{1}+1+2+(p-1) \text {. }
$$

We prove a better result: namely, the order of $\mathbf{u}$ is

$$
\left(n_{1}+n_{1}+1+2\right) p
$$

and we give an example to show that this is best possible (see $§ 5$ ).

There are substantial new difficulties to overcome. In the strictly hyperbolic case, piecewise smooth initial data give rise to a piecewise smooth solution. Rauch and Reed use this fact to reduce many proofs to studying the propagation of jumps along the singularity bearing curves. In the nonstrictly hyperbolic case this is no longer possible, even if the initial data are piecewise smooth. The solution will have unbounded derivatives near the outgoing characteristic from $P$. This is not merely a technical difficulty, but a basic feature of nonstrictly hyperbolic problems. (See $[4,5$, 6].) Moreover, the higher order derivatives of $\mathbf{u}$ will not even be $L^{1}$ near these characteristics. This poses a major problem, since we wish to express derivatives of the components of $\mathbf{u}$ as integrals over backward characteristics. We overcome this difficulty by observing that, near each characteristic, only certain directional derivatives of certain components of $\mathbf{u}$ blow up. We develop a calculus for recursively proving this fact and using it to show that in the integrals over backward characteristics, the high order singularities cancel out.

2. Existence of the solution. Throughout this paper we consider only local properties of the solution near the curve of degeneracy and, without loss of 
generality, our system can be cast in the form:

$$
\begin{aligned}
& X_{i} u(x, t)=f_{i}(x, t, \mathbf{u}), \quad i=1, \ldots, n ; \quad \mathbf{u}(x, 0)=\mathbf{u}^{0}(x), \\
& \text { where } X_{i} \text { denotes the vector field } \partial_{t}+\lambda_{i}(x, t) \partial_{x}, \text { and } \\
& \lambda_{1}(x, t)=\alpha(x, t)(t-\bar{t})^{p-1} ; \quad \lambda_{2}(x, t)=0 .
\end{aligned}
$$

We may assume that $\bar{t}$ is a fixed positive number, $\alpha(x, t) \geqslant \alpha_{1}>0$, and $\lambda_{i}, f_{i}$, and $\alpha$ are smooth functions. We consider $\mathbf{u}$ only in a trapezoidal region $R_{T}$ whose boundary is composed on the left by the curve tangent at each point to the characteristic vector field of maximal speed, on the right by the curve tangent at each point to the characteristic vector field of minimal speed, and above and below by the lines $t=T(T>\bar{t})$ and $t=0$.

The vector fields $X_{1}$ and $X_{2}$ are parallel on the line $t=\bar{t}$, and we impose the following nontangency conditions on the remaining characteristic vector fields:

$$
\begin{aligned}
\inf _{(x, t) \in R_{T}}\left|\lambda_{i}(x, t)-\lambda_{j}(x, t)\right| \geqslant \delta>0 \text { for } i, j= & 1, \ldots, n, \\
& i>j,(j, i) \neq(1,2) .
\end{aligned}
$$

We shall use the following terminology: $x=\gamma_{i}\left(t ; x_{0}, t_{0}\right)$ represents the $i$ th characteristic passing through $\left(x_{0}, t_{0}\right)$. The characteristics through $(0, \bar{t})$ play a special role in the sequel and are denoted by $C_{i}$ (our argument is local and we can assume that every $\gamma_{i}\left(t ; x_{0}, t_{0}\right)$ exists in $\left.R_{T}\right)$. The functions $\gamma_{i}$ can be inverted for $i=$ $3,4, \ldots, n$, while for $i=1$ we can denote the two branches of the inverse function by $t=\gamma_{1,+}^{-1}\left(x ; x_{0}, t_{0}\right)\left(\gamma_{1,+}^{-1}\left(x ; x_{0}, t_{0}\right) \geqslant \bar{t}\right)$ and $t=\gamma_{1,-}^{-1}\left(x ; x_{0}, t_{0}\right)\left(\gamma_{1,-}^{-1}\left(x ; x_{0}, t_{0}\right)<\bar{t}\right)$. We also use the space $C_{*}^{m}\left(R_{T} \backslash S\right)$ of $R^{n}$-valued functions on $R_{T} \backslash S$ which are $m$ times continuously differentiable on $R_{T} \backslash S$ and all the first $m$ partial derivatives have continuous extensions to the boundaries.

In this section we prove the existence of a solution of (2.1) in an appropriate space, involving derivatives of order $n_{1}+2$, when we choose the initial data $\mathbf{u}^{0}$ such that

$$
\begin{aligned}
& u_{1}^{0} \in C_{*}^{\infty}\left(\mathbb{R} \backslash\left\{\gamma_{1}(0,0, \bar{t})\right\}\right) \cap C^{n_{1}}(\mathbb{R}), \\
& u_{2}^{0} \in C_{*}^{\infty}(\mathbb{R} \backslash\{0\}) \cap C^{n_{2}}(\mathbb{R}) ; \quad u_{i}^{0} \in C^{\infty}(\mathbb{R}) \quad \forall i>2 .
\end{aligned}
$$

In the sequel we always assume $n_{2} \geqslant n_{1}$. The other case can be treated likewise after the change of coordinates $(x, t) \rightarrow(y, t)$, where $y=\gamma_{1}(\bar{t} ; x, t)$, that reverses the role of $C_{1}$ and $C_{2}$.

We will look for the solution of our system as a fixed point, in an appropriate space of vector functions, of the map

$$
(\mathscr{M} \mathbf{u})_{i}(x, t)=u_{i}^{0}\left(\gamma_{i}(0 ; x, t)\right)+\int_{0}^{t} f_{i}\left(\mathbf{u}\left(\gamma_{i}(r ; x, t), r\right), \gamma_{i}(r ; x, t), r\right) d r
$$

In fact, if $\mathbf{w}=\mathscr{M} \mathbf{w}$, then $\mathbf{w}$ satisfies (2.1) in the sense of distributions and takes on the correct initial data. The space of functions that we must choose has a rather complicated definition because we must allow certain derivatives of order $n_{1}+2$ to be unbounded, but integrable, near $C_{1}$ and $C_{2}$ for $t \geqslant \bar{t}$; at the same time all the derivatives are bounded everywhere for $t<\bar{t}$. In fact if we start with a jump of order $n$ travelling on $C_{1}$ and a jump of order $n_{2}$ travelling on $C_{2}$, we expect the following 
picture to be true: For $t<\bar{t}$, where the system is strictly hyperbolic, the jump discontinuities propagate along the characteristics $C_{1}$ and $C_{2}$; for $t>\bar{t}$ the derivatives of order $n_{1}+1$ still exhibit only jump discontinuities (on $C_{1}$ if $n_{2}>n_{1}$, on $C_{1}$ and $C_{2}$ if $n_{2}=n_{1}$ ), but certain derivatives of order $n_{1}+2$ will behave like (distance from $C_{1}$ or $\left.C_{2}\right)^{-1+1 / p}$; hence, they are unbounded but integrable (see (2.5)(ii), (a), (b)); however, some derivatives of order $n_{1}+2$ still exhibit only jump discontinuities (see (2.5)(iii)), and this feature will be basic in the smoothness results of $\S \S 3$ and 4. More precisely, we define $F^{n_{1}, n_{2}}$ to be the space of vector functions $\mathbf{u}=\left\{u_{i}\right\}_{i=1}^{n}$ such that

(i) $u_{1} \in C^{n_{1}}\left(R_{T}\right) \cap C_{*}^{n_{1}+1}\left(R_{T} \backslash C_{1}\right)$;

$$
u_{2} \in \begin{cases}C^{n_{1}+1}\left(R_{T}\right) & \text { if } n_{1}>n_{2}, \\ C^{n_{1}}\left(R_{T}\right) \cap C_{*}^{n_{1}+1}\left(R_{T} \backslash C_{2}\right) & \text { if } n_{1}=n_{2}\end{cases}
$$

(ii) $\partial_{x}^{n_{1}+2} u_{i} \in C\left(R_{T} \backslash\left(C_{1} \cup C_{2}\right)\right) \quad$ if $i=1,2$.

Moreover, if $\Omega_{i}, i=1, \ldots, 4$, are the open regions of Figure 2.1, the following estimates hold, with $y(x, t)=\gamma_{1}(\bar{t} ; x, t)$ (see Figure 2.1):

(a) $\left.\left.\sup _{(x, t) \in \Omega_{2}}\left|\partial_{x}^{n_{1}+2} u_{1}(x, t)\right||\bar{t}-t+| y(x, t)\right|^{1 / p}\right|^{p-1}$

$$
t<\bar{i}
$$

$$
\begin{aligned}
& \quad+\sup _{(x, t) \in \Omega_{1}}\left|\partial_{x}^{n_{1}+2} u_{1}(x, t)\right|+\sup _{(x, t) \in \cup_{i=2}^{4} \Omega_{i}}\left|\partial_{x}^{n_{1}+2} u_{1}(x, t)\right||y(x, t)|^{1-1 / p} \\
& \equiv M_{1}(u)<+\infty
\end{aligned}
$$

(b)

$$
\begin{aligned}
\sup _{\substack{(x, t) \in \Omega_{4} \\
t<i}} & \left.\left.\left|\partial_{x}^{n_{1}+2} u_{2}\right||\bar{t}-t+| x\right|^{1 / p}\right|^{p-1} \\
& +\sup _{(x, t) \in \Omega_{2} \cup \Omega_{3} \cup \Omega_{4}}\left|\partial_{x}^{n_{1}+2} u_{2}\right||x|^{1-1 / p}+\sup _{(x, t) \in \bar{\Omega}_{1}}\left|\partial_{x}^{n_{1}+2} u_{2}\right| \\
& \equiv M_{2}(u)<+\infty .
\end{aligned}
$$

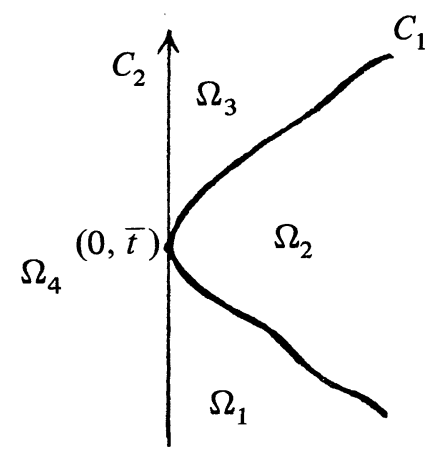

FIGURE 2.1 
(iii)

$$
\begin{gathered}
\left(\prod_{n} X_{1}^{\alpha_{n}} \partial_{x}^{\beta_{n}}\right) u_{1} \in C_{*}\left(R_{T} \backslash\left(C_{1} \cup C_{2}\right)\right), \\
\sum\left(\alpha_{n}+\beta_{n}\right)=n_{1}+2, \quad \sum \alpha_{n} \geqslant 1, \\
\partial_{t}^{a} \partial_{x}^{n_{1}+2-q} u_{2} \in C_{*}\left(R_{T} \backslash\left(C_{1} \cup C_{2}\right)\right) \text { if } 1 \leqslant q \leqslant n_{1}+2 ; \\
u_{i} \in \begin{cases}C^{n_{1}+1}\left(R_{T}\right) \cap C_{*}^{n_{1}+2}\left(R_{T} \backslash C_{1}\right) & \text { if } n_{1}>n_{2}, i=3, \ldots, n, \\
C^{n_{1}+1}\left(R_{T}\right) \cap C_{*}^{n_{1}+2}\left(R_{T} \backslash\left(C_{1} \cup C_{2}\right)\right) & \text { if } n_{1}=n_{2} ; \\
u_{i}(x, 0)=u_{i}^{0} .\end{cases}
\end{gathered}
$$

We are now ready to state the existence theorem:

THEOREM 2.1. Let $R_{T}$ be a region of $\mathbb{R}^{2}$ as described in the introduction. If the initial data $\mathbf{u}^{0}$ satisfies conditions (2.3) then there is a unique solution of problem (2.1) in the space $F^{n_{1}, n_{2}}$.

The crucial step is to show that $\mathscr{M}$ takes $F^{n_{1}, n_{2}}$ into itself. It is relatively straightforward, using Lemma 2.1 of [2], to show that $\mathscr{M} \mathbf{u}$ satisfies conditions (2.5)(i), (iii), (v).

We will now prove (ii)(b) for $(\mathscr{M} \mathbf{u})_{2}$. The proof of (ii)(a) is obtained in the same way after having "straightened out" the first vector field with the change of coordinates $\phi:(x, t) \rightarrow(y, t), y=\gamma_{1}(\bar{t} ; x, t)$. The vector field $X_{1}$ is in fact mapped in $\phi X_{1}=(0,1)$ and $X_{2}$ in $X_{2}=\left(-(t-\bar{t})^{p-1} \tilde{\alpha}(y, t), 1\right)$. We thus obtain the same type of geometry with a reversed role for $X_{1}$ and $X_{2}$. For $x \neq 0$ all derivatives of order $n_{1}+2$ of any $\mathbf{u}$ in $F^{n_{1}, n_{2}}$ are integrable functions along any $i$ th characteristic, $i \geqslant 1$ (see Lemma 2.2); to compute $\partial_{x}^{n_{1}+2} u_{2}$ we can use formula (2.4), taking the derivative under the integral sign.

$$
\begin{aligned}
\partial_{x}^{n_{1}+2}(\mathscr{M} \mathbf{u})_{2}(x, t)= & \partial_{x}^{n_{1}+2} u^{0}(x)+\int_{0}^{t} \partial_{x}^{n_{1}+2} f_{2}(\mathbf{u}(x, r), x, r) d r \\
+ & \lambda_{1}^{-1}\left(x, \gamma_{1,-}^{-1}(x ; 0, \bar{t})\right)\left[\partial_{x}^{n_{1}+1} f_{2}(\mathbf{u})\right]_{\left\langle x, \gamma_{1,-}^{-1}(x ; 0, \bar{t})\right\rangle} \\
& \cdot \chi\left\{(x, t): x>0, t>\gamma_{1,-}^{-1}(x ; 0, \bar{t})\right\} \\
& +\lambda_{1}^{-1}\left(x, \gamma_{1,+}^{-1}(x ; 0, \bar{t})\right)\left[\partial_{x}^{n_{1}+1} f_{2}(\mathbf{u})\right]_{\left\langle x, \gamma_{1,+}^{-1}(x ; 0, \bar{t})\right\rangle} \\
& \cdot \chi\left\{(x, t): x>0, t>\gamma_{1,+}^{-1}(x ; 0, \bar{t})\right\},
\end{aligned}
$$

where $[\cdot]_{\left\langle x, \gamma_{i}\right\rangle}$ denotes the jump across $C_{i}$ from right to left. Using its definition, we can easily prove the following expression for $\gamma_{1}(t ; y, \bar{t})$ :

$$
\begin{aligned}
& \gamma_{1}(t ; \gamma, \bar{t})=y+(t-\bar{t})^{p} G(y, t) \\
& G(y, t)=\int_{\bar{t}}^{t} \frac{(r-\bar{t})^{p-1}}{(t-\bar{t})^{p}} \alpha\left(\gamma_{1}(r ; \gamma, \bar{t}), r\right) d r .
\end{aligned}
$$

As $\alpha_{2}>\alpha(x, t)>\alpha_{1}>0$ in $R_{T}$, then $\alpha_{3}>G(y, t)>\alpha_{4}>0$. Moreover,

$$
c_{1} x^{-1+1 / p} \leqslant\left|\lambda_{1}^{-1}\left(x, \gamma_{1,+}^{-1}(x ; 0, \bar{t})\right)\right| \leqslant c_{2} x^{-1+1 / p} \text {. }
$$


Using these inequalities and (2.5)(ii) to estimate the derivatives of $\mathbf{u}$, we get from (2.6) the following bound on $\partial_{x}^{n_{1}+2}(\mathscr{M} \mathbf{u})_{2}(x, t)$ for every $(x, t)$ in $\Omega_{2} \cup \Omega_{3} \cup \Omega_{4}$, $t>\bar{t}$ :

$$
\begin{aligned}
& \left|\partial_{x}^{n_{1}+2}(\mathscr{M} \mathbf{u})_{2}(x, t)\right| \leqslant c_{1}\left(1+x^{-1+1 / p}+\int_{\gamma_{1,-}^{-1}(x ; 0, \bar{t})}^{t}[y(x, r)]^{-1+1 / p} d r\right) \\
& \leqslant c_{2}\left(1+x^{-1+1 / p}+\int_{\gamma_{1,-}^{-1}(x)}^{T}\left|x-(r-\bar{t})^{p} G(y(x, r), r)\right|^{-1+1 / p} d r\right) \\
& \quad \leqslant c_{3}\left(1+x^{-1+1 / p}+\int_{x}^{T}|x-q|^{-1+1 / p} q^{-1+1 / p} d q\right) \\
& \quad \leqslant c_{4}\left(1+x^{-1+1 / p}+x^{-1+2 / p} \int_{0}^{T}(1-s)^{-1+1 / p} s^{-1+1 / p} d s\right) \\
& \quad \leqslant c_{5}\left(1+x^{-1+1 / p}\right),
\end{aligned}
$$

where we have used (2.7) to express $y(x, r)$.

A similar proof gives the desired bound in region $\Omega_{4}$ for $t<\bar{t}$. The proof of (2.5)(iv) is based on the following computational lemma that insures that $\partial_{x}^{n_{1}+2} u_{i}$, $i=1,2$, are integrable functions along any $j$ th characteristic, $j \geqslant 3$.

LeMmA 2.2. Let $y(x, t)=\gamma_{1}(\bar{t} ; x, t)$. Then the two functions $\gamma_{j}(r ; x, t)^{-1+1 / p}$ and $\left[y\left(\gamma_{j}(r ; x, t), r\right)\right]^{-1+1 / p}$ are integrable in the r-variable everywhere in $[0, T]$ and $\forall(x, t) \in R_{T}$ if $j \geqslant 3$.

Proof. We give the proof for the second function; the other can be treated similarly.

Let $t_{j}(x, t)$ be the second coordinate of the intersection between $\gamma_{j}(r ; x, t)$ and $C_{1} \cdot y(x, t)$ is zero for any point $(x, t) \in C_{1}$, hence $y\left(\gamma_{j}(r ; x, t), r\right)=0$ when $r=t_{j}(x, t)$. We have to expand $y\left(\gamma_{j}(r ; x, t), r\right)$ in Taylor series near $t_{j}(x, t)$. As $y(x, t)=\gamma_{1}(\bar{t} ; x, t)$ we get

$$
\begin{aligned}
& y\left(\gamma_{j}(r ; x, t), r\right)=\left(r-t_{j}\right)\left[\partial_{3} \gamma_{1}\left(\bar{t} ; \gamma_{j}(h ; x, t), h\right)\right. \\
& \left.\quad+\partial_{2} \gamma_{1}\left(\bar{t} ; \gamma_{j}(h ; x, t), h\right) \lambda_{j}\left(\gamma_{j}(h ; x, t), h\right)\right]\left.\right|_{h=t_{j}+\mathscr{I}\left(r-t_{j}\right)}
\end{aligned}
$$

$$
\mathscr{I} \in(0,1)
$$

where

$$
\partial_{i} \gamma_{j}\left(x_{1} ; x_{2}, x_{3}\right)=\partial_{x_{i}} \gamma_{j}\left(x_{1} ; x_{2}, x_{3}\right), \quad i=1,2,3 .
$$

Our function $\left[y\left(\gamma_{j}(r ; x, t), r\right)\right]^{-1+1 / p}$ will be integrable if we prove that the quantity inside brackets is bounded away from zero.

Recalling (2.7), we have

$$
x=\gamma_{1}(\bar{t} ; x, t)+(t-\bar{t})^{p} G\left(\gamma_{1}(\bar{t} ; x, t), t\right),
$$

which implies

$$
\begin{aligned}
& \partial_{2} \gamma_{1}(\bar{t} ; x, t)=\frac{1}{1+(t-\bar{t})^{p} \partial_{1} G\left(\gamma_{1}(\bar{t} ; x, t), t\right)}, \\
& \partial_{3} \gamma_{1}(\bar{t} ; x, t)=\frac{-\lambda_{1}}{1+(t-\bar{t})^{p} \partial_{1}\left(\gamma_{1}(\bar{t} ; x, t), t\right)},
\end{aligned}
$$


as $\partial_{t}\left[(t-\bar{t})^{p} G(y, t)\right]=\lambda_{1}$, by the definition of $G$. The bracketed quantity then equals

$$
\left.\frac{\lambda_{j}-\lambda_{1}}{1+(t-\bar{t})^{p} \partial_{1} G}\left(\gamma_{j}(h ; x, t), h\right)\right|_{h=t_{1}(x, t)+I\left(r-t_{j}\right)},
$$

which is bounded away from zero for the nontangency condition (2.2).

We can now prove (2.5)(iv) for $(\mathscr{M} \mathbf{u})_{j}, j \geqslant 3$, by just differentiating (2.4) and applying Lemma 2.2. Let $y_{j}=\gamma_{j}(\bar{t} ; x, t)$. Then

$$
\begin{aligned}
\partial_{x}^{n_{1}+2}(\mathscr{M} \mathbf{u})_{j}(x, t)= & {\left[\left(\partial_{2} \gamma_{j}\left(0 ; y_{j}(x, t), \bar{t}\right) \partial_{x} y_{j}(x, t) \partial_{q}\right)^{n_{1}+2} u_{j}^{0}(q)\right] } \\
& \cdot \gamma_{j}(0 ; x, t)+\int_{0}^{t}\left[\left(\partial_{2} \gamma_{j}\left(r ; \gamma_{j}(x, t), \bar{t}\right) \partial_{x} y_{j}(x, t) \partial_{x}\right)^{n_{1}+2} f_{j}\right] \\
& \cdot\left(\mathbf{u}, \gamma_{j}\left(r ; y_{j}(x, t), \bar{t}\right), r\right) d r \\
+ & \partial_{x} t_{j}(x, t)\left[\left(\partial_{2} \gamma_{j}\left(t_{i} ; y_{j}, \bar{t}\right) \partial_{x} y_{j} \partial_{x}\right)^{n_{1}+1} f_{j}\right]_{\left\langle\gamma_{1}\left(t_{j}(x, t) ; 0, \bar{t}\right) ; t_{j}(x, t)\right\rangle}
\end{aligned}
$$

The term $\partial_{x} t_{j}(x, t)$ is shown to be bounded taking the derivative of the equality defining $t_{j}, \gamma_{j}\left(t_{j}(x, t) ; x, t\right)=\gamma_{1}\left(t_{j}(x, t) ; 0, \bar{t}\right)$, which gives

$$
\left|\partial_{x} t_{j}(x, t)\right|=\left|\frac{\partial_{2} \gamma_{j}\left(t_{j}(x, t) ; x, t\right)}{\lambda_{1}-\lambda_{j}}\right|<c_{1} \quad \text { as } j \geqslant 3 .
$$

Estimate (2.5)(iv) follows immediately from (2.5)(ii), (2.11), (2.12), and Lemma 2.2. Thus, $\mathscr{M} \mathbf{u} \in F^{n_{1}, n_{2}}$ if $\mathbf{u} \in F^{n_{1}, n_{2}}$. To solve $\mathscr{M} \mathbf{u}=\mathbf{u}$ we show that $\mathscr{M}$ is a contraction on a ball in $F^{n_{1}, n_{2}}$ in a suitable norm. Define

$$
\begin{aligned}
\|\mathbf{u}\|_{t_{0}}= & \sum_{i=1}^{n}\left\|u_{i}\right\|_{C_{*}^{n_{1}+1}\left(R_{T} \backslash\left(C_{1} \cup C_{2}\right)\right), t_{0}} \\
& +\varepsilon_{1}\left[\tilde{M}_{1}^{t_{0}}(\mathbf{u})+\tilde{M}_{2}^{t_{0}}(\mathbf{u})+\sum_{i=3}^{n}\left\|u_{i}\right\|_{C_{*}^{n_{1}+2}\left(R_{T} \backslash\left(C_{1} \cup C_{2}\right)\right), t_{0}}\right],
\end{aligned}
$$

where

$$
\left\|u_{i}\right\|_{C_{*}^{k}\left(R_{T} \backslash\left(C_{1} \cup C_{2}\right)\right), t_{0}} \sum_{s=1}^{k} \sum_{0}^{s} \sup _{\substack{(x, t) \in R_{T} \backslash\left(C_{1} \cup C_{2}\right) \\ t \leqslant \bar{t}_{0}}}\left|\left(\partial_{x}\right)^{l}\left(\partial_{t}\right)^{s-l} u_{i}(x, t)\right|
$$

and

$$
\tilde{M}_{i}^{t_{0}}(\mathbf{u})=M_{i}^{t_{0}}(\mathbf{u})+\sup _{\substack{(x, t) \in R_{T} \backslash\left(C_{1} \cup C_{2}\right) \\ t \leqslant t_{0}}} \sum_{l=0}^{n_{1}+1}\left|\left(\partial_{x}\right)^{l}\left(X_{i}\right)^{n_{1}+2-l} u_{i}\right|, \quad i=1,2 .
$$

$M_{i}^{t_{0}}$ is defined as in (2.5)(iii), adding the condition $t \leqslant t_{0}$, and $\varepsilon_{1}$ is a positive constant that will be chosen shortly. We first show that $\mathscr{M}$ maps a ball in $F^{n_{1}, n_{2}}$ of radius $2\left\|\mathbf{u}^{0}\right\|_{t_{0}}$ into itself if $t_{0}$ is small enough. The two basic tools are Lemma 2.2 and the estimates

$$
\int_{0}^{t}(y(x, r))^{-1+1 / p} d r \leqslant c_{1} x^{-1+2 / p}
$$


and

$$
\int_{0}^{t}\left(\gamma_{1}(r ; x, t)\right)^{-1+1 / p} d r \leqslant c_{2}(y(x, t))^{-1+2 / p}
$$

obtained in (2.8). From the definition (2.13) of $\|\cdot\|_{t_{0}}$, it is clear that the only term requiring some attention is

$$
\varepsilon_{1}\left[\tilde{M}_{1}^{t_{0}}(\mathscr{M} \mathbf{u})+\tilde{M}_{2}^{t_{0}}(\mathscr{M} \mathbf{u})+\sum_{i=3}^{n}\left\|u_{i}\right\|_{C_{*}^{n_{1}+2}}\right] .
$$

We show how to bound $\tilde{M}_{2}^{t_{0}}(\mathscr{M} \mathbf{u})$; the other two terms can be treated likewise. Let $\|\mathbf{u}\|_{t_{0}} \leqslant 2\left\|\mathbf{u}^{0}\right\|_{t_{0}} ;$ then

$$
\begin{aligned}
& \varepsilon_{1} \tilde{M}_{2}^{t_{0}}(\mathscr{M} \mathbf{u}) \leqslant 2 c_{1} t_{0}\|u\|_{t_{0}} \\
& +\varepsilon_{1} \sup _{(x, t) \in R_{T}}\left\{|x|^{1-1 / p}\left|\lambda_{1}^{-1}\right|\left(x, \gamma_{1,-}^{-1}(x ; 0, t)\right)\left[\partial_{x}^{n_{1}+1} f_{2}\right]_{\left\langle x, \gamma_{1,-}^{-},(x)\right\rangle}\right. \\
& +|x|^{1-1 / p}\left|\lambda_{1}^{-1}\right|\left(x, \gamma_{1,+}^{-1}(x)\right)\left[\partial_{x}^{n_{1}+1} f_{2}\right]_{\left\langle x, \gamma_{1,+}^{-1}(x)\right\rangle} \\
& \left.+\int_{t}^{t_{0}} \sum_{i=1}^{2}\left|\partial_{x}^{n_{1}+2} u_{i} \partial_{i} f_{2}\right|(x, r)|x|^{1-1 / p} d r\right\} \\
& \leqslant c_{2}\left\{t_{0}\|\mathbf{u}\|_{t_{0}}+\varepsilon_{1}\|\mathbf{u}\|_{C_{*}^{n_{1}+1}+}+t_{0} M_{2}^{t_{0}}(\mathbf{u})\right. \\
& \left.+M_{1}^{t_{0}}(\mathbf{u}) \int_{t}^{t_{0}}|x|^{1-1 / p}|y(x, r)|^{-1+1 / p} d r\right\} \\
& \leqslant c_{3}\left(t_{0}+\varepsilon_{1}\right)\|\mathbf{u}\|_{t_{0}} \leqslant c_{4}\left(t_{0}+\varepsilon_{1}\right)\left\|\mathbf{u}^{0}\right\|_{t_{0} .} .
\end{aligned}
$$

Choosing $\varepsilon_{1}$ and $t_{0}$ small enough, one gets $\|\mathscr{M} \mathbf{u}\|_{t_{0}} \leqslant 2\left\|\mathbf{u}^{0}\right\|_{t_{0}}$. The proof that $\mathscr{M}$ is a contraction follows the same lines. Of the various terms of $\|\mathscr{M} \mathbf{u}-\mathscr{M} \mathbf{w}\|_{t_{0}}$ we show only how to bound $\varepsilon_{1} \tilde{M}_{2}^{t_{0}}(\mathscr{M} \mathbf{u}-\mathscr{M} \mathbf{w})$, the remaining ones being similar.

$$
\begin{aligned}
& \varepsilon_{1} \tilde{M}_{2}^{t_{0}}(\mathscr{M} \mathbf{u}-\mathscr{M} \mathbf{w}) \leqslant \varepsilon_{1}|x|^{1-1 / p}\left|\lambda_{1}^{-1}\right|\left(x, \gamma_{1,-}^{-1}(x)\right)\left[\left|\partial_{x}^{n_{1}+1} f_{2}(\mathbf{u})-\partial_{x}^{n_{1}+1} f_{2}(\mathbf{w})\right|\right]_{\left\langle x, \gamma_{1}^{-1},(x)\right\rangle} \\
&+ \varepsilon_{1}|x|^{1-1 / p}\left|\lambda_{1}^{-1}\right|\left(x, \gamma_{1,+}^{-1}(x)\right)\left[\left|\partial_{x}^{n_{1}+1}\left(f_{2}(\mathbf{u})-f_{2}(\mathbf{w})\right)\right|\right]_{\left\langle x, \gamma_{1}^{-1},(x)\right\rangle} \\
&+ \sup _{(x, t) \in R_{T}} \int_{0}^{t_{0}} \varepsilon_{1}|x|^{-1+1 / p}\left|\partial_{x}^{n_{1}+2}\left(f_{2}(\mathbf{u})-f_{2}(\mathbf{w})\right)\right|(x, r) d r \\
& \leqslant c_{1}\left\{\varepsilon_{1}\|\mathbf{u}-\mathbf{w}\| C_{*}^{n_{1}+1}\left(R_{T} \backslash\left(C_{1} \cup C_{2}\right)\right)+\sup _{(x, t) \in R_{T}} \int_{0}^{t_{0}} \sum_{i=1}^{2} \varepsilon_{1}|x|^{1-1 / p}\right. \\
&\left.\cdot\left|\partial_{i} f_{2}(\mathbf{u}) \partial_{x}^{n_{1}+2} u_{i}-\partial_{i} f_{2}(\mathbf{w}) \partial_{x}^{n_{1}+2} w_{i}\right|(x, r) d r+t_{0} 2\left\|\mathbf{u}^{0}\right\|_{t_{0}}\|\mathbf{u}-\mathbf{w}\|_{t_{0}}\right\}
\end{aligned}
$$




$$
\begin{aligned}
& \leqslant c_{2}\left\{\left(\varepsilon_{1}+t_{0}\right)\left\|\mathbf{u}^{0}\right\|_{t_{0}}\|\mathbf{u}-\mathbf{w}\|_{t_{0}}\right. \\
&+\sup _{(x, t) \in R_{T}}\left[\int _ { 0 } ^ { t _ { 0 } } \sum _ { i = 1 } ^ { 2 } \left[\left|\partial_{i} f_{2}(\mathbf{w})\right|\left|\left(\partial_{x}^{n_{1}+2} u_{i}-\partial_{x}^{n_{1}+2} w_{i}\right)\right| \varepsilon_{1}|x|^{1-1 / p}\right.\right. \\
&\left.\left.\quad+\left|\partial_{x}^{n_{1}+2} u_{i}\right| \varepsilon_{1}|x|^{-1+1 / p}\left|\left(\partial_{i} f_{2}(\mathbf{u})-\partial_{i} f_{2}(\mathbf{w})\right)\right|\right](x, r) d r\right] \\
&\left.\leqslant c_{3} x\left(\varepsilon_{1}+t_{0}\right)\left\|\mathbf{u}^{0}\right\|_{t_{0}}\|\mathbf{u}-\mathbf{w}\|_{t_{0}}\right\} .
\end{aligned}
$$

This shows the existence of a solution $\mathbf{u} \in F^{n_{1}, n_{2}}$ for a small time; one would like to apply this local existence theorem finitely many times to get existence in all $R_{T}$. For this purpose one must prove a priori bounds on the solution $\mathbf{u}$ to be sure that the interval of existence does not shrink to zero. This can be accomplished, following the scheme of $\S 2$ of [2], using estimates like (2.8) and (2.11). The proof is trivial and will be omitted.

3. Smoothness of the solution. In the previous section we have shown that if the initial data satisfy conditions (2.3), then the system we are considering has a classical solution in $R_{T} \backslash\left(C_{1} \cup C_{2}\right)$ even if $n_{1}=-1$, the case corresponding to a jump discontinuity in $\mathbf{u}_{1}^{0}$. However, certain derivatives of order $n_{1}+2$ of $\mathbf{u}$ blow up near $C_{1}$ and $C_{2}$. When one tries to take more derivatives of $\mathbf{u}$ using formula (2.4), one soon finds that the functions involved are no longer integrable unless one makes a very careful choice of the differential operators appearing under the integral sign. More precisely, we are going to show that derivatives like $X_{i}^{k} u_{J}$ are bounded near $C_{i}$, away from $(0, \bar{t})$, for $i, j=1, \ldots, n$ and $\forall k$. This will permit us to find an explicit representation of the derivatives of any order of $\mathbf{u}$ in $R_{T} \backslash\left(\bigcup_{i=1}^{n} C_{i}\right)$ again using the map $\mathscr{M}$, provided that only the differential operator $X_{i}$ appears in the portion of integral crossing $C_{i}$. This allows us to set up a bootstrap argument to prove that the solution $\mathbf{u}$ is actually in $C^{\infty}\left(R_{T} \backslash\left(\bigcup_{i=1}^{n} C_{i}\right)\right)$.

THEOREM 3.1. Let $\mathbf{u}$ be a solution of system (2.1) with initial data $\mathbf{u}^{0}$ satisfying the regularity conditions (2.3). Then $\mathbf{u} \in C^{\infty}\left(R_{T} \backslash\left(\bigcup_{i=1}^{n} C_{i}\right)\right)$.

Proof. Let $D^{a} u_{i}$ be a derivative of $u_{i}$ of order $a$. Suppose by induction that

(3.1) (i) $\mathbf{u} \in\left[C^{n_{1}+1+k}\left(R_{T} \backslash\left(\bigcup_{i=1}^{n} C_{i}\right)\right)\right]^{n}$,

(ii) $X_{i}^{h} D^{n_{1}+1} u_{J}$ is continuous across $C_{i} \backslash\{(0, \bar{t})\}$ if $i \neq j, h \leqslant k ; X_{i}^{h} D^{n_{1}+1} u_{i}$ is continuous up to $C_{i} \backslash\{(0, \bar{t})\}, h \leqslant k$.

We postpone the proof of the case $k=1$, and we start assuming (3.1) to be true for $k$. Using the system we get that derivatives like

$$
\prod_{i, q_{i}} X_{i}^{q_{i}} D^{n_{1}+1} X_{J}^{r} u_{J}
$$

(where $i=1, \ldots, n, \quad \sum_{1}^{n} a_{i}+r=k+1, r \geqslant 1$ ) exist and are continuous in $R_{T} \backslash\left(\cup_{i=1}^{n} C_{i}\right)$, and this implies the existence and the continuity, in the same region, of

$$
X_{J}^{r} \prod_{i, q_{i}} X_{i}^{q_{i}} D^{n_{1}+1} u_{J}
$$




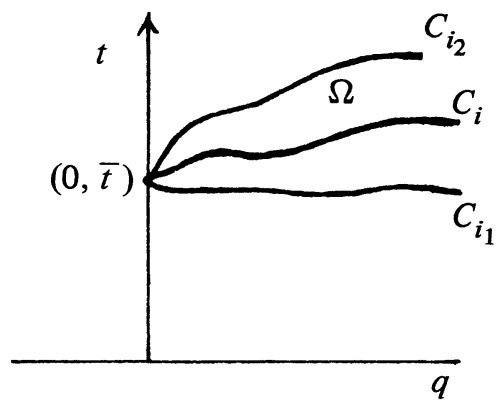

FIGURE 3.1

Moreover, we have that

$$
X_{J}^{k+1} D^{n_{1}+1} u_{J}=X_{J}^{k} \tilde{D}^{n_{1}+1} u_{J}+X_{J}^{k} D^{n_{1}+1} f_{J}(\mathbf{u})
$$

clearly satisfies, by the inductive hypothesis at step $k$, the desired properties near $C_{J}$. Let us now fix the index $j$ of the component of the solution. We can straighten out the $j$ th characteristic vector field by applying the change of coordinates $\phi_{J}:(x, t) \rightarrow$ $(q, t)$, where we have set $q=\gamma_{J}(\bar{t} ; x, t)$. For simplicity we will again call $X_{i}, \lambda_{i}, f_{i}$ the vector fields and the functions of the new variables corresponding to $X_{i}, \lambda_{i}, f_{i}$. The map $\phi_{J}$ preserves the nontangency conditions (2.2) for the vector fields different from $X_{1}$ and $X_{2}$. The region $R_{T}$ is subdivided by the characteristics $C_{i}$ into various subregions. Let us focus on one of them. The situation is sketched in Figure 3.1.

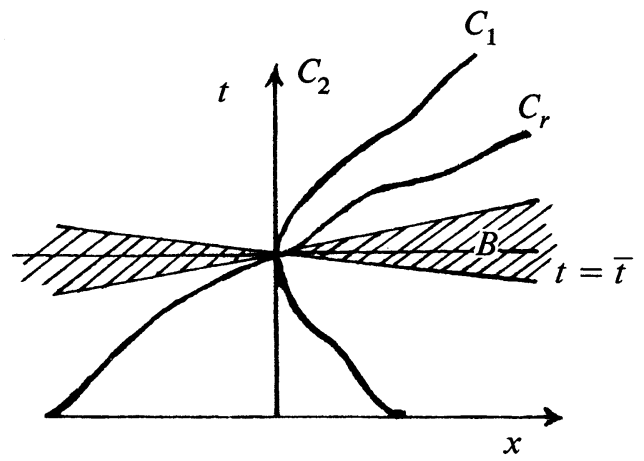

FIGURE 3.2

We assume that the derivatives of order $k+1$ of $u_{j}$ exist below $C_{i}$, and we prove they also exist in the open region $\Omega$ between $C_{i_{2}}$ and $C_{i} . C_{i_{1}}$ is there only to pick an initial value for $u_{J}$. We can also assume that no other characteristic $C_{r}$ lies between $C_{i}, C_{i_{1}}, C_{i_{2}}$. The argument we present works everywhere in $R_{T}$ if $j \geqslant 3$. If $j=1,2$, owing to the tangency of the vector fields $X_{1}$ and $X_{2}$, we can use it only outside a region $B$ containing the line $t=\bar{t}$, but no $C_{r}$, as in Figure 3.2. The proof that $u_{i} \in C^{\infty}(B)$ for $i=1,2$ is trivial and will be sketched at the end of this section.

To show that $u_{J}$ is in $C^{k+1}(\Omega)$, we need only prove the existence and continuity across $C_{i}$ of $X_{i}^{k+1} D^{n_{1}+1} u_{J}$, as we already know the existence of the derivatives of order $n_{1}+1+k+1$ containing at least one differentiation along the direction $X_{J}$ 
(in the open region that we are considering, $X_{i}$ and $X_{J}$ cannot be parallel). We can also assume $D^{n_{1}+1}=\partial_{q}^{n_{1}+1}$, as we can write any direction as $a \partial_{q}+b X_{J}$, and the terms containing $X_{J}$ are continuous by the previous argument. The following formula for the change of order of differentiation holds:

$$
\begin{aligned}
X_{r} X_{J}^{k} F(x, t)=\left\{X_{J}^{k} X_{r}-\sum_{s=0}^{k-1} c_{s}^{k}\left[X_{J}^{s+1}\left(\frac{\lambda_{r}-\lambda_{J}}{\partial_{2} \gamma_{J}\left(t ; y_{J}(x, t), \bar{t}\right)}\right)\right]\right. \\
\left.\cdot X_{J}^{k-s-1}\left(\frac{\partial_{2} \gamma_{J}\left(t ; y_{J}(x, t), \bar{t}\right)}{\lambda_{r}-\lambda_{J}}\right)\left(X_{r}-X_{J}\right)\right\} F(x, t) \\
\quad(r \neq j),
\end{aligned}
$$

where $c_{0}^{0}=1, c_{s}^{k+1}=c_{s}^{k}+\left(\begin{array}{l}k \\ s\end{array}\right), 0<s \leqslant k, c_{k+1}^{k+1}=1$. Using the previous formula and recalling that in the $(q, t)$-plane $X_{J}$ is parallel to $\partial t$, we have

$$
\begin{aligned}
X_{J} X_{i}^{k} \partial_{q}^{n_{1}+1} u_{J} & =X_{i}^{k} \partial_{q}^{n_{1}+1} X_{J} u_{J}+\sum_{s=0}^{k-1} c_{s}^{k}\left[X_{i}^{s+1}\left(\lambda_{i}(q, t)\left(\partial_{2} \gamma_{i}\left(t ; y_{i}(q, t), \bar{t}\right)\right)^{-1}\right)\right] \\
& \cdot \sum_{l=0}^{k-s-1}\left[X_{i}^{k-s-1-l} \partial_{2} \gamma_{i}\left(t ; y_{i}(q, t), \bar{t}\right) \lambda_{i}^{-1}(q, t)\right] X_{i}^{l}\left(X_{i}-X_{J}\right) \partial_{q}^{n_{1}+1} u_{J} \\
= & \left\{\partial_{J} f_{J}(\mathbf{u})+X_{i}\left[\log \frac{\lambda_{i}(q, t)}{\partial_{2} \gamma_{i}\left(t ; y_{i}(q, t), \bar{t}\right)}\right]\right\} X_{i}^{k} D^{n_{1}+1} u_{J} \\
& +F_{J, k-1}(\mathbf{u})+\sum_{\substack{r \neq j \\
r=1,2, \ldots, n}}\left(\partial_{r} f_{J}\right) X_{i}^{k} D^{n_{1}+1} u_{r},
\end{aligned}
$$

where $F_{J, k-1}$ contains only derivatives like $X_{i}^{a} D^{n_{1}+1} u, a \leqslant k-1$. Formula (3.3) makes sense since $\lambda_{i}>\alpha_{J}>0$ if $i \neq j$ (because we are in $R_{T} \backslash\left(C_{1} \cup C_{2}\right)$ and because of conditions (2.2)). Using (3.3) and the continuity of $X_{i}^{k} \partial_{q}^{n_{1}+1} u_{j}$ across $C_{i}$, we get the following explicit expression for $\left(X_{i}^{k} \partial_{q}^{n_{1}+1} u_{j}\right)(q, t)$ :

$$
\begin{aligned}
&\left(X_{i}^{k} \partial_{q}^{n_{1}+1} u_{j}\right)(q, t) \\
&=\exp \left(M_{j}(q, t)\right)\left\{\left(X_{i}^{k} \partial_{q}^{n_{1}+1} u_{j}\right)\left(q, t_{b}(q)\right)\right. \\
&\left.+\int_{t_{b}(q)}^{t} e^{-M_{j}(q, s)}\left[\sum_{\substack{r=1 \\
r \neq j}}^{n}\left(\partial_{r} f_{j}\right) X_{i}^{k} \partial_{q}^{n_{1}+1} u_{r}+F_{j, k-1}\right](q, s) d s\right\}
\end{aligned}
$$

where

$$
M_{j}(q, t)=\int_{t_{b}(q)}^{t}\left[\partial_{j} f_{j}(\mathbf{u})+X_{i}\left(\log \left(\frac{\lambda_{i}(q, r)}{\partial_{2} \gamma_{i}\left(r ; y_{i}(q, t), \bar{t}\right)}\right)\right)\right] d r
$$

and

$$
t_{b}(q)=b \gamma_{i}(q ; 0, t)+(1-b) \gamma_{i_{1}}(q ; 0, t), \quad b \in(0,1)
$$


For $(q, t)$ in $\Omega$ we can try to compute $X_{i}^{k+1} \partial_{q}^{n_{1}+1} u_{j}(q, t)$ by differentiating the r.h.s. of (3.4). Formally, we obtain

$$
\begin{aligned}
& X_{i}^{k+1} \partial_{q}^{n_{1}+1} u_{j}(q, t)=\left(X_{i} M_{j}\right)(q, t)\left(X_{i}^{k} \partial_{q}^{n_{1}+1} u_{j}\right)(q, t)+\exp \left(M_{j}(q, t)\right) \\
& \cdot\left\{X_{i}\left(X_{i}^{k} \partial_{q}^{n_{1}+1} u_{j}\right)\left(q, t_{b}(q)\right)\right. \\
&\left.\quad+X_{i} \int_{t_{b}(q)}^{t} e^{-M_{j}(q, s)}\left[\sum_{\substack{r=1 \\
r \neq j}}^{n} \partial_{r} f_{j} X_{i}^{k} \partial_{q}^{n_{1}+1} u_{r}+F_{j, k-1}\right](q, s) d s\right\} .
\end{aligned}
$$

The only term on the r.h.s. of (3.5) that must be shown to be well defined is the derivative of the integral, as the other ones involve only derivatives of order at most $n_{1}+1+k$ in $\Omega$ and derivatives of order $n_{1}+1+k+1$ at $\left(q, t_{b}(q)\right)$ where they are known to exist. To compute the derivative of the integral we use a procedure consisting of creating under the integral sign the differential operator $X_{r}$ in front of $\partial_{r} f_{j} X_{i}^{k} \partial_{q}^{n_{1}+1} u_{r}$ (because the derivatives that are created have already been shown to exist) and $X_{i}$ in front of $F_{j, k-1}$.

$$
\begin{aligned}
& X_{i} \int_{t_{b}(q)}^{t}\left[\partial_{r} f_{j}(\mathbf{u}) X_{i}^{k} \partial_{q}^{n_{1}+1} u_{r}\right] e^{-M_{j}}(q, s) d s \\
& =\lambda_{i}(q, t) \lim _{\Delta q \rightarrow 0} \frac{1}{\Delta q}\left\{\int_{t_{b}(q+\Delta q)}^{\gamma_{i}^{-1}(q+\Delta q ; s, t)}\left[e^{-M_{j}} \partial_{r} f_{j} X_{i}^{k} \partial_{q}^{n_{1}+1} u_{r}\right](q+\Delta q, s) d s\right. \\
& \left.-\int_{t_{b}(q)}^{t}\left[e^{-M_{j}} \partial_{r} f_{j} X_{i}^{k} \partial_{q}^{n_{1}+1} u_{r}\right](q, s) d s\right\} .
\end{aligned}
$$

We break up the path of integration in several parts according to the scheme in Figure 3.3 (we have supposed that $C_{r}$ is steeper than $C_{i}$, but the same argument works for the other case):

$\left(q, \gamma_{r}^{-1}(q ; q+\Delta q\right.$,

$(q, t)$

$\left(q, \gamma_{i}^{-1}(q ; 0, \bar{t})\right)$

$\left(q, \gamma_{r}^{-1}(q ; q+\Delta q\right.$,

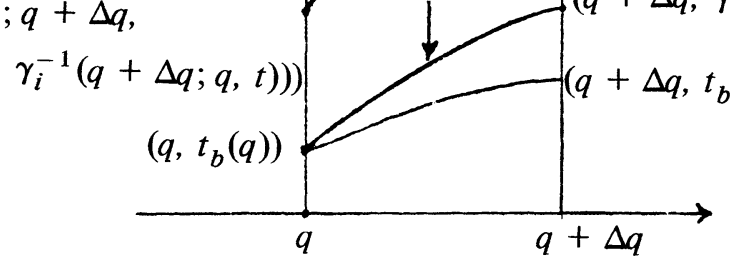

Figure 3.3 
Let $\exp \left(-M_{j}\right) \partial_{r} f_{j} X_{i}^{k} \partial_{q}^{n_{1}+1} u_{r}=U_{r}$. Then the r.h.s. of (3.6) can be written as

$$
\begin{aligned}
& \lambda_{i}(q, t) \lim _{\Delta q \rightarrow 0} \frac{1}{\Delta q} \int_{t_{b}(q+\Delta q)}^{\gamma_{r}^{-1}\left(q+\Delta q ; q, t_{b}(q)\right)} U_{r}(q+\Delta q, s) d s \\
& +\left[\int_{\gamma_{r}^{-1}\left(q+\Delta q ; q, t_{b}(q)\right)}^{\gamma_{r}^{-1}(q+\Delta q ; 0, \bar{t})} U_{r}(q+\Delta q, s) d s\right. \\
& \left.\quad-\int_{t_{b}(q)}^{\gamma_{r}^{-1}\left(q ; q+\Delta q, \gamma_{i}^{-1}(q+\Delta q ; 0, \bar{t})\right)} \cdot U_{r}(q, s) d s\right] \\
& +\int_{\gamma_{i}^{-1}(q+\Delta q ; 0, \bar{t})}^{\gamma_{r}^{-1}\left(q+\Delta q: q, \gamma_{i}^{-1}(q ; 0, \bar{t})\right)} U_{r}(q+\Delta q, s) d s \\
& -\int_{\gamma_{r}^{-1}\left(q ; q+\Delta q, \gamma_{i}^{-1}(q+\Delta q ; 0, \bar{t})\right)}^{\gamma_{i}^{-1}(q ; 0, \bar{t})} U_{r}(q, s) d s \\
& +\left[\int_{\gamma_{i}^{-1}\left(q+\Delta q ; q, \gamma_{i}^{-1}(q ; 0, \bar{t})\right)}^{\gamma_{i}^{-1}(q+\Delta q ; 0, \bar{t})} U_{r}(q+\Delta q, s) d s\right. \\
& \left.\quad-\int_{\gamma_{i}^{-1}(q ; 0, \bar{t})}^{\gamma_{r}^{-1}\left(q ; q+\Delta q, \gamma_{i}^{-1}(q+\Delta q ; q, t)\right)} U_{r}(q, s) d s\right] \\
& -\int_{\gamma_{r}^{-1}\left(q ; q+\Delta q, \gamma_{i}^{-1}(q+\Delta q ; q, t)\right)}^{t} U_{r}(q, s) d s .
\end{aligned}
$$

In the first integral inside each bracket we use the change of variables

$$
s(l)=\gamma_{r}^{-1}(q+\Delta q ; q, l),
$$

whose Jacobian is given by $J(q+\Delta q, q, l)=\partial_{3} \gamma_{r}^{-1}(q+\Delta q ; q, l)$, where $J(q, q, l)=1$ since

$$
\begin{aligned}
J(q, q, l) & =\lim _{\Delta l \rightarrow 0} \frac{\gamma_{r}^{-1}(q ; q, l+\Delta l)-\gamma_{r}^{-1}(q ; q, l)}{\Delta l} \\
& =\lim _{\Delta l \rightarrow 0} \frac{1}{\Delta l}(l+\Delta l-l)=1 .
\end{aligned}
$$

Hence,

$$
\begin{gathered}
\lim _{\Delta q \rightarrow 0} \frac{1}{\Delta q}[J(q+\Delta q, q, l)-1]=\lim _{\Delta q \rightarrow 0} \frac{1}{\Delta q}[J(q+\Delta q, q, l)-J(q, q, l)] \\
=\left(\partial_{1} \partial_{3} \gamma_{r}^{-1}\right)(q ; q, l)=\left(\partial_{3} \partial_{1} \gamma_{r}^{-1}\right)(q ; q, l)=\partial_{l} \lambda_{r}^{-1}(q, l) .
\end{gathered}
$$

The limit for $\Delta q \rightarrow 0$ of the sum of the two square brackets is then equal to

$$
\begin{gathered}
\lim _{\Delta q \rightarrow 0} \frac{1}{\Delta q} \int_{t_{b}(q)}^{\gamma_{r}^{-1}\left(q: q+\Delta q, \gamma_{i}^{-1}(q+\Delta q: q, t)\right)}\left[U_{r}\left(q+\Delta q, \gamma_{r}^{-1}(q+\Delta q ; q, l)\right)\right. \\
\left.\cdot J(q+\Delta q, q, l)-U_{r}(q, l)\right] d l \\
=\int_{t_{b}(q)}^{t}\left[\lambda_{r}^{-1}(q, l) X_{r} U_{r}+\left(\partial_{t} \lambda_{r}^{-1}\right) U_{r}\right](q, l) d l
\end{gathered}
$$


where we have used equality (3.8). The integrand in the r.h.s. of (3.9) is continuous and integrable. In fact, using (3.2) we get

$$
\begin{aligned}
& X_{r} U_{r}=X_{r}\left(e^{-M_{j}} \partial_{r} f_{j} X_{i}^{k} \partial_{q}^{n_{1}+1} u_{r}\right) \\
& =\left[X_{r}\left(e^{-M_{j}} \partial_{r} f_{j}\right)\right] X_{i}^{k} \partial_{q}^{n_{1}+1} u_{r} \\
& +e^{-M_{j}} \partial_{r} f_{j}\left\{X_{i}^{k} X_{r} \partial_{q}^{n_{1}+1} u_{r}+\sum_{s=0}^{k-1} C_{s}^{k}\left[X_{i}^{s+1} a\right] X_{j}^{k-s-1} a^{-1} \partial_{q}^{n_{1}+1} u_{r}\right\} \\
& =\left[X_{r}\left(e^{-M_{j}} \partial_{r} f_{j}\right)\right] X_{i}^{k} \partial_{q}^{n_{1}+1} u_{r}+e^{-M_{j}} \partial_{r} f_{j} X_{i}^{k} D^{n_{1}+1} f_{r}+R_{k-1},
\end{aligned}
$$

where $a(a, t)=\left(\lambda_{r}-\lambda_{i}\right)\left(\partial_{2} \gamma_{i}\left(t ; \gamma_{i}(q, t), \bar{t}\right)\right)^{-1}$ and $R_{k}$ contains only derivatives of order $n_{1}+1+k$ that exist up to $C_{i} ; a$ and $a^{-1}$ are $C^{\infty}(\Omega)$ functions for the usual restrictions (2.2) on the vector fields. Moreover, $X_{r}\left(e^{-M_{j}} \partial_{r} f_{j}\right)$ contains only first order derivatives that are at least integrable.

The limit of the remaining terms on the r.h.s. of (3.7) is easily taken, and the result is the following explicit formula.

LEMMA 3.1. For every $(q, t)$ in $\Omega$ we have

$$
\begin{aligned}
& \left(X_{i}^{k+1} \partial_{q}^{n_{1}+1} u_{j}\right)(q, t)=\left(X_{i} M_{j}\right)\left(X_{i}^{k} \partial_{q}^{n_{1}+1} u_{j}\right)(q, t) \\
& +e^{M_{j}(g, t)}\left\{X_{i}\left(X_{i}^{k} \partial_{q}^{n_{1}+1} u_{j}\right)\left(q, t_{b}(q)\right)\right. \\
& +\int_{t_{b}(q)}^{t} \sum_{\substack{r=1 \\
r \neq j}}^{n}\left(\lambda_{r}^{-1} X_{r} U_{r}+\left(\partial_{t} \lambda_{r}^{-1}\right) U_{r}\right)(q, s) d s \\
& +\int_{t_{b}(q)}^{t}\left[\lambda_{i}^{-1} X_{i}\left(e^{-M_{j}} F_{j, k-1}\right)+\left(\partial_{t} \lambda_{i}^{-1}\right)\left(e^{-M_{j}} F_{j, k-1}\right)\right](q, s) d s \\
& +\sum_{\substack{r=1 \\
r \neq j}}^{n}\left[\left(\lambda_{r}^{-1}\left(q, t_{b}(q)\right)-\partial_{q} t_{b}(q)\right) U_{r}\left(q, t_{b}(q)\right)\right. \\
& \left.\left.\quad+\left[U_{r}\left(\lambda_{i}^{-1}-\lambda_{r}^{-1}\right)\right]_{\left\langle q, \gamma_{i}^{-1} k(q, 0, i)\right\rangle}+\left(U_{r}\left(\lambda_{r}^{-1}-\lambda_{r}^{-1}\right)\right)(q, t)\right]\right\}
\end{aligned}
$$

One can repeat the same argument in the region above $C_{j_{2}}$ and then for the other components of $\mathbf{u}$.

$X_{i}^{k+1} \partial_{q}^{n_{1}+1} u_{j}$ computed as above seems to have a jump discontinuity across $C_{i}$. This depends only on the technique we had to use in order to avoid the creation of certain derivatives of order $n_{1}+1+k+1$ inside the integrals. But once we know that $X_{i}^{k+1} \partial_{q}^{n_{1}+1} u_{r}$ exists continuously up to $C_{i}$ for $r=1, \ldots, n$, we can repeat our argument, taking, inside the integrals, derivatives along the $i$ th direction on all the functions. This eliminates the jump across $C_{i}$. The previous argument does not work 
for $u_{i}, i=1,2$, in region $B$ of Figure 3.3. We fix $\varepsilon>0$ and define

$$
B_{\varepsilon}=B \cap\{(x, t): y(x, t)>\varepsilon \text { if } t>\bar{t}\} \cap\{(x, t): x>\varepsilon \text { if } t>\bar{t}\},
$$

where $y(x, t)=\gamma_{1}(\bar{t} ; x, t)$.

$B_{\varepsilon}$ has a role similar to $R_{T}$, if we draw the first or second backward characteristic from any point in $B_{\varepsilon}$ it will intersect $\partial B_{\varepsilon} \cap \partial B$, that we can consider as a noncharacteristic initial line. We want to prove that $\forall \varepsilon>0, u_{i}, i=1,2$, are in $C^{k+1+n_{1}+1}\left(B_{\varepsilon}\right)$, knowing that $u_{i} \in C^{k+1+n_{1}+1}$ below $B_{\varepsilon}$ and $u_{r} \in C^{k+1+n_{1}+1}\left(R_{T}\right)$ if $r=3, \ldots, n$. We can consider $u_{r}, r \geqslant 3$, as fixed $C^{k+1+n_{1}+1}$ functions and need only prove that the semilinear nonstrictly hyperbolic system $X_{i} u_{i}=F_{i}, i=1,2$, with initial data in $C^{k+1+n_{1}+1}(\partial B)$ and $F_{i} \in C^{k+1+n_{1}+1}\left(B_{\varepsilon}\right)$, has a solution in the same space. This can be accomplished by a straightforward contraction map argument that we omit.

The case $k=1$ could be treated, if $n_{1} \geqslant 0$, in exactly the same way, also using the definition of $F^{n_{1}, n_{2}}$. But if $n_{1}=-1$, we cannot use the trick of linearizing in the higher order derivative as in (3.3). We then have to use a less intuitive procedure. As $\mathbf{u}$ is in $F^{n_{1}, n_{2}}$, we need only show that $X_{1} D^{n_{1}+1} u_{j}$ is continuous across $C_{1} \backslash\{(0, \bar{t})\}$ for $j>1$ (if $n_{1}=n_{2}$ we must repeat the same proof for $C_{2}$ ). We consider $X_{1} D^{n_{1}+1} u_{2}$; the other components can be treated similarly. The only interesting case is $D^{n_{1}+1}=$ $\partial_{x}^{n_{1}+1}$, because otherwise we can use the system to lower the order of the derivatives. We have the following expressions for the jumps of $\partial_{t} \partial_{x}^{n_{1}+1} u_{2}$ and $\partial_{x}^{n_{1}+2} u_{2}$ across the two branches of $C_{1}$ :

$$
\begin{aligned}
& {\left[\partial_{t} \partial_{x}^{n_{1}+1} u_{2}\right]_{\left\langle x, \gamma_{1}^{-1}(x ; 0, i)\right\rangle}=\left[\partial_{x}^{n_{1}+1} f_{2}(\mathbf{u})\right]_{\substack{\left\langle x, \gamma_{1}^{-1}(x ;-) \\
(-)\right.}}} \\
& {\left[\partial_{x} \partial_{x}^{n_{1}+1} u_{2}\right]_{\left\langle x, \gamma_{1,+}^{-1}(x ; 0, \bar{t})\right\rangle}=-\left(\partial_{x} \gamma_{1,+}^{-1}(x ; 0, \bar{t})\right)\left[\partial_{x}^{n_{1}+1} f_{2}(\mathbf{u})\right]_{\left\langle x, \gamma_{1,+}^{-1}(x ; 0, \bar{t})\right\rangle}} \\
& =-\lambda_{1}^{-1}\left(x, \gamma_{1,+(-)}^{-1}(x ; 0, \bar{t})\right)\left[\partial_{x}^{n_{1}+1} f_{2}(\mathbf{u})\right]_{\substack{\left\langle x, \gamma_{1, i}^{-1}(x ; 0, \bar{t})\right\rangle \\
(-)}}^{(-)} .
\end{aligned}
$$

From (3.11) and (3.12) we get

$$
\left[X_{1} \partial_{x}^{n_{1}+1} u_{2}\right]_{\substack{\left\langle x, \gamma_{1,+}^{1}(-) \\(-)\right.}}=\left[\partial_{t} \partial_{x}^{n_{1}+1} u_{2}\right]_{\substack{\left\langle x, \gamma_{1,+}^{1},-(x)\right\rangle \\(-)}}^{1}+\left[\lambda_{1} \partial_{x}^{n_{1}+2} u_{2}\right]_{\substack{\left\langle x, \gamma_{1,-}^{1}(-) \\(-)\right.}}=0 .
$$

4. Smoothness across $C_{j}, j \geqslant 3$. We can now consider the regularity of the solution $\mathbf{u}$ of our system across the characteristics $C_{j}, j \geqslant 3$. If the system were linear the solution would be smooth across them; in the semilinear case we prove instead that $\mathbf{u}$ is at least of order $\left(n_{1}+n_{1}+1+2\right) p$ on $C_{j}$ for $j \geqslant 3$. The counterexample in the last section shows that this is the best possible result in the general case. The proof is quite long, and we subdivide it into various steps.

Step 1. $\mathbf{u} \in C^{2\left(n_{1}+1\right)}\left(R_{T} \backslash\left(C_{1} \cup C_{2}\right)\right),\left(\partial_{t} X_{1}\right)^{n_{1}+1} u_{j} \in C\left(R_{T}\right), j=3, \ldots, n$.

We prove Step 1 by induction. We know already from $\S 2$ that $u_{j} \in C^{n_{1}+1}\left(R_{T}\right)$ if $j \geqslant 3$ and $u_{i} \in C_{*}^{n_{1}+1}\left(R_{T} \backslash C_{i}\right)$ if $i=1,2$. If $n_{1}=-1$ there is nothing to prove; hence, we assume $n_{1} \geqslant 0$. This enables us to use the same procedure as in $\S 3$. More precisely, let $L^{s}$ be the differential operator defined by

$$
L^{s}= \begin{cases}\left(\partial_{t} X_{1}\right)^{s / 2} & \text { if } s \text { is even } \\ X_{1}\left(\partial_{t} X_{1}\right)^{(s-1) / 2} & \text { if } s \text { is odd }\end{cases}
$$


We assume by induction that $\mathbf{u} \in C^{n_{1}+1+k}\left(R_{T} \backslash\left(C_{1} \cup C_{2}\right)\right), L^{n_{1}+1+k} u_{j} \in C\left(R_{T}\right)$ $\forall j \geqslant 3, k \geqslant 0$, and we want to prove an analogous statement for $k+1$. Any derivative of $u_{j}$ of order $n_{1}+1+k$ containing a differentiation along the direction $X_{j}$ can be shown to be continuous using the system and the inductive hypothesis. Hence, we need only prove that $L^{n_{1}+1+k+1} u_{j} \in C\left(R_{T}\right)$, as $\partial_{t}$ (or $\left.X_{1}\right)$ and $X_{j}$ are nonparallel vector fields. Following the same procedure of $\S 3$, we consider the linear equation satisfied by $L^{n_{1}+1+k} u_{j}$, write an integral formula for its solution (not containing $L^{n_{1}+1+k} u_{j}$ ), and then compute $L^{n_{1}+1+k+1} u_{j}$ with it, being careful to create the right kind of derivatives inside the integrals involved. We first need a formula for $X_{j} L^{n_{1}+1+k} u_{j}$ in terms of derivatives like $L^{s} u_{j}$, if $j \geqslant 3, s \leqslant n_{1}+1+k$, and $\partial_{x}^{s} u_{i}$ for $i=1,2, s \leqslant n_{1}+1$. The following formula is easily proven by induction:

$$
\begin{aligned}
X_{j} L^{n_{1}+1+k} u_{j} & =h_{\alpha}(\mathbf{u}, x, t) \prod_{j}\left\{\partial_{x}^{\alpha_{j}^{1}} u_{1} \partial_{x}^{\alpha_{j}^{2}} u_{2} \prod_{i} L^{\alpha_{j}^{i}} u_{i}\right\}(t-\bar{t})^{l\left(\left|\alpha^{1}\right|+\left|\alpha^{2}\right|, n_{1}+1+k\right)}, \\
\alpha^{i} & =\left\{\alpha_{i}^{i}, \ldots, \alpha_{n_{1}+1+k}^{i}\right\}, \quad i=1, \ldots, n, i \geqslant 3,
\end{aligned}
$$

with conditions

$$
\max _{j} \alpha_{j}^{2} \leqslant\left[\frac{n_{1}+1+k+1}{2}\right], \quad \max _{j} \alpha_{j}^{1} \leqslant\left[\frac{n_{1}+1-k}{2}\right],
$$

where $h_{\alpha}$ are $C^{\infty}$ functions of their arguments and $l(m, n)$ is given by

$$
l(m, n)=\max (m p-n, 0) .
$$

The different bounds on $\max _{j}\left(\alpha_{j}^{1}, \alpha_{j}^{2}\right)$ depends on the order in which we take the derivatives in $L^{n_{1}+1+k}$. The idea is that a derivative like $\partial_{t} u_{1}$ can be written as $X_{1} u_{1}-\lambda_{1} \partial_{x} u_{1}$, while any derivative of $u_{j}$ is a linear combination, with $C^{\infty}$ coefficients in $R_{T}$, of $\partial_{t}$ (or $X_{1}$ ) and $X_{j}$.

We can then follow the scheme of $\S 3$ step by step: using (4.2) we can write a formula similar to (3.4) for $L^{n_{1}+1+k} u_{j}$. To compute $L^{n_{1}+1+k+1} u_{j}$ we can use the procedure of Lemma 3.1. Namely, inside the various integrals corresponding to the various terms of the sum in the r.h.s. of (4.2), we create (with a change of variable similar to the one used before) a differential operator according to the following rule:

$X_{i}$ in front of $L^{n_{1}+1+k} u_{i}, i \geqslant 3, i \neq j, \partial_{t}$ (or $X_{1}$ according to the parity of $\left.n_{1}+1+k\right)$ in front of the remaining ones.

The terms, analogous to the ones in (3.10), coming from the segment near $C_{1}$ (or $\left.C_{2}\right)$ and $(x, t)$ either vanish or are continuous, as all the terms of the integrand are in $C\left(R_{T}\right)$ if $k<n_{1}$. There is one exception: the case $k=n_{1}$; then a term like $\partial_{x}^{n_{1}+1} u_{2}$ appears in the integrand, but the next derivative is $\partial_{t}$, so that in computing the derivative there is no jump term across $C_{2}$ (as $\partial_{t}$ is parallel to $C_{2}$ ), and the jump across $C_{1}$ is zero since $\partial_{x}^{n_{1}+1} u_{2} \in C_{*}\left(R_{T} \backslash C_{2}\right)$. We omit the details referring to $\S 3$. Once we know that $\left(\partial_{t} X_{1}\right)^{n_{1}+1} u_{j} \in C\left(R_{T}\right) \forall j \geqslant 3$, we can write a formula for $\left(\partial_{t} X_{1}\right)^{n_{1}+1} u_{j}$ that is the true achievement of Step 1. Using (4.2) for $k=n_{1}+1$ we 
have

$$
\begin{aligned}
& \left(\partial_{t} X_{1}\right)^{n_{1}+1} u_{j}(x, t) \\
& =e^{M_{j}(x, t)}\left\{I_{2\left(n_{1}+1\right)}\left(\mathbf{u}^{0}\left(\gamma_{j}^{-1}(0 ; x, t)\right)\right)\right. \\
& \quad+\int_{\gamma_{j}^{-1}(0, x, t)}^{x}\left[\frac{e^{-M_{j}}}{\lambda_{j}}\left(\sum_{s, r=0}^{n_{1}+1}(t-\bar{t})^{l\left(s+r, 2\left(n_{1}+1\right)\right)} \partial_{x}^{s} u_{1} \partial_{x}^{r} u_{2} L^{s, r}+R_{1}\right)\right] \\
& \left.\cdot\left(q, \gamma_{j}^{-1}(q ; x, t)\right) d q\right\},
\end{aligned}
$$

where

$$
\begin{aligned}
& \text { (i) } L^{s, r}=\sum_{\alpha_{i=1, \ldots n}^{\prime}} \quad h_{\alpha}(\mathbf{u}, x, t)(t-\bar{t}) l^{\left(\left|\alpha^{1}\right|+\left|\alpha^{2}\right|, 0\right)} \\
& \alpha^{i}=\left\{\alpha_{1}^{i}, \ldots, \alpha_{2\left(n_{1}+1\right)}^{i}\right\} \\
& \sum_{1^{i}}^{n}\left|\alpha^{i}\right| \leqslant 2\left(n_{1}+1\right)-(r+s) \\
& \alpha_{n}^{1} \geqslant s ; \alpha_{n}^{2} \leqslant r ; \alpha_{n}^{1} \leqslant 2\left(n_{1}+1\right)-1, i \geqslant 3 \\
& \cdot \prod_{n}\left(\prod_{i \geqslant 3} L^{\alpha_{n}^{i}} u_{i}\right) \partial_{x}^{\alpha_{n}^{1}} u_{1} \partial_{x}^{\alpha_{n}^{2}} u_{2}
\end{aligned}
$$

(ii) $R_{1}=\sum_{i=3}^{n} h_{i}(\mathbf{u}, x, t) L^{2\left(n_{1}+1\right)} u_{i} ; \quad M_{j}=\int_{0}^{x} m_{j}\left(\mathbf{u}, q, \gamma_{j}^{-1}(q ; x, t)\right) d q$.

$I_{2\left(n_{1}+1\right)}\left(\mathbf{u}^{0}\right)$ is a $C^{\infty}$ function depending on $\partial_{x}^{r} \mathbf{u}^{0}$ for $r<2\left(n_{1}+1\right)$, and $m, H_{\alpha}, h_{i}$ are $C^{\infty}$ functions of their arguments. In (4.4) the various singular terms are separated, and each will be treated in a different way.

REMARK 4.1. Formula (4.4) does not make sense if $n_{1}=-1$. That case is, however, much easier. In fact, one of the major problems if $n_{1} \geqslant 0$ is the term $R_{1}$, as there is no higher order derivative of $u_{j}, j \geqslant 3$, that is bounded or integrable near $(0, \bar{t})$. Instead, if $n_{1}=-1, X_{1}^{p} u_{j}$ and $\partial_{t}^{p} u_{j}$ are bounded in certain regions containing $(0, \bar{t})$. We will explain how to deal with the case $n_{1}=-1$ later on.

In computing higher order derivatives of $u_{j}$ using (4.7), we create inside the integral derivatives like $X_{i}^{k} \partial_{x}^{n_{1}+1} u_{r}, i, r=1,2$, whose behavior we must now investigate. We set up an inductive argument that will show, at the same time, the bounds on such derivatives and the continuity across $C_{j}, j \geqslant 3$, of the derivatives of $\mathbf{u}$ up to the order $\left(n_{1}+n_{1}+1+2\right) p$. For this purpose we must study in great detail the behavior of certain derivatives of the solution near the "bad" characteristics $C_{1}$ and $C_{2}$.

We assume the following inductive hypothesis for

$$
n_{1}+2 \leqslant k \leqslant\left(n_{1}+n_{1}+1+2\right) p:
$$

(4.6) All the derivatives of order $k$ of the solution $\mathbf{u}$ are in the space

$$
C\left(R_{T} \backslash\left(C_{1} \cup C_{2}\right)\right) \text {. }
$$




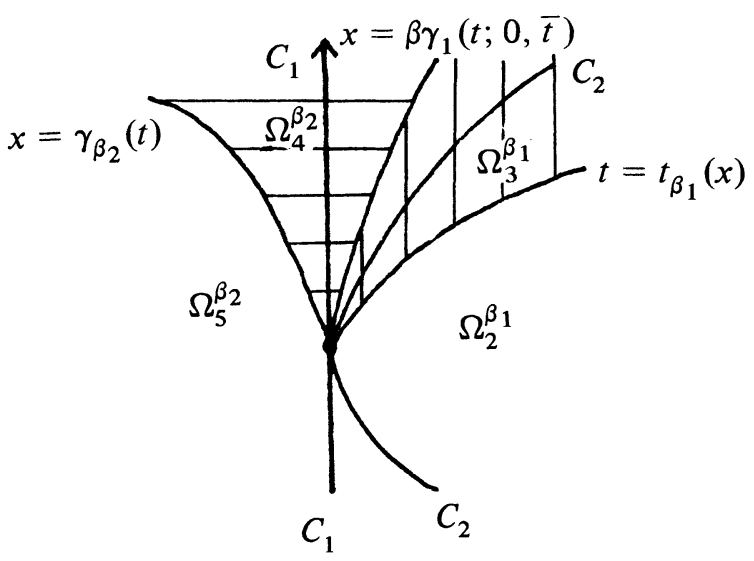

FIGURE 4.1

Moreover, the following bounds are true $\forall \beta \in(0,1)$ (see Figure 4.1):

(i)

$$
\begin{gathered}
\left|X_{1}^{k-s} \partial_{x}^{s} u_{r}\right|(x, t) \leqslant M_{1}\left[1+x^{-(k-s) / p-s+n_{1}+1+1 / p}\right], \quad r=1, \ldots, n, \\
s= \begin{cases}0, \ldots, k & \text { if }(x, t) \in \Omega_{2}^{\beta_{1}} \equiv\left\{x>0, \gamma_{1,-}^{-1}(x ; 0, \bar{t}) \leqslant t \leqslant t \beta_{1}(x)\right\}, \\
0, \ldots, n+1 & \text { if }(x, t) \in \Omega_{3}^{\beta_{1}} \equiv\left\{x>0, t_{\beta_{1}}(x) \leqslant t \leqslant \gamma_{\beta}^{-1}(x)\right\},\end{cases}
\end{gathered}
$$

where $t_{\beta_{1}}(x)=\beta_{1} \gamma_{1,+}^{-1}(x ; 0, \bar{t})+\left(1-\beta_{1}\right) \bar{t}$ and $t=\gamma_{\beta}^{-1}(x)$ is the upper branch of the inverse of the function $x=\beta \gamma_{1}(t ; 0, \bar{t})$. (The two regions are sketched in Figure 4.1.)

(ii)

$$
\begin{gathered}
\quad\left|\partial_{t}^{k-s} \partial_{x}^{s} u_{r}\right|(x, t) \leqslant M_{2}\left[1+(y(x, t))^{-(k-s) / p-s+n_{1}+1+1 / p}\right], \quad r=1, \ldots, n, \\
s= \begin{cases}0, \ldots, k & \text { if }(x, t) \in \Omega_{3}^{\beta_{2}} \equiv\left\{x<0, t \leqslant \gamma_{\beta_{2}}^{-1}(x)\right\}, \\
0, \ldots, n+1 & \text { if }(x, t) \in \Omega_{4}^{\beta_{3}} \equiv\left\{x<0, t \geqslant \gamma_{\beta_{2}}^{-1}(x) \text { or } x>0, t \geqslant \gamma_{\beta}^{-1}(x)\right\},\end{cases}
\end{gathered}
$$

where $\gamma_{\beta_{2}}^{-1}(x)$ is the upper branch of the inverse function of the integral curve through $(0, \bar{t})$ of the vector field $\left(-\beta_{2} \lambda_{1}, 1\right)$ for $\beta_{2} \in(0,1)$.

(iii) If $j \geqslant 3$ and $k \geqslant 2\left(n_{1}+1\right)$, then

$$
\begin{aligned}
& \sup _{(x, t) \in \Omega_{2}^{\beta_{1}} \cup \Omega_{3}^{\beta_{1}}}\left|X_{1}^{k-2\left(n_{1}+1\right)} L^{2\left(n_{1}+1\right)} u_{j}(x, t)\right| \\
& \leqslant M_{3}\left(1+x^{-\left(k-2\left(n_{1}+1\right)-1\right) / p+\left(n_{1}+2\right)(1-1 / p)}\right),
\end{aligned}
$$

and

$$
\begin{aligned}
& \sup _{(x, t) \in \Omega_{5}^{\beta_{2}} \cup \Omega_{4}^{\beta_{2}}}\left|\partial_{t}^{k-2\left(n_{1}+1\right)} L^{2\left(n_{1}+1\right)} u_{j}(x, t)\right| \\
& \quad \leqslant M_{4}\left(1+(y(x, t))^{-\left(k-2\left(n_{1}+1\right)-1\right) / p+\left(n_{1}+2\right)(1-1 / p)}\right) .
\end{aligned}
$$

Step 2. Proofs of the bounds on $X_{i}^{k-s} \partial_{x}^{s} u_{j}, i, j=1,2$.

We suppose the inductive hypothesis (4.6) true for $k$. We explicitly show the bound for $X_{1}^{k-s} \partial_{x}^{s} u_{i}$, as $\partial_{t}^{k-s} \partial_{x}^{s} u_{i}$ can be treated likewise after the usual change of coordinates that exchanges the role of $\partial_{t}$ and $X_{i}$. 
We first consider a bound for $\partial_{x}^{k+1} u_{i}, i=1,2$ and $k \leqslant\left(n_{1}+n_{1}+1+2\right) p$ in the region $\Omega_{2}^{\beta_{1}}=\left\{(x, t): x>0, t \leqslant t_{\beta_{1}}(x)\right\}$. The problem is that the line $t=\bar{t}$ lies in this region, where $X_{1}$ and $\partial_{t}$ are parallel. This hinders us from using the procedure of $\S 3$.

However, recalling equalities (2.7), we have in $\Omega_{2}^{\beta_{1}}$ :

$$
\begin{aligned}
y(x, t) & =x-G(y(x, t), t)(t-\bar{t})^{p} \geqslant c_{1}\left(x-\left(t_{\beta_{1}}(x)-\bar{t}\right)^{p} G(y(x, t), t)\right) \\
& =c_{1} x\left[1-\beta_{1}^{p} G(y, t)\left(G\left(y\left(x, \gamma_{1,+}^{-1}(x ; 0, t)\right), \gamma_{1,+}^{-1}(x ; 0, \bar{t})\right)\right)^{-1}\right] \geqslant c_{2} x
\end{aligned}
$$

if $\beta_{1} \in(0,1)$ is small enough and $t \geqslant \bar{t}$.

$$
\begin{aligned}
\left(\bar{t}-t+y^{1 / p}(x, t)\right) & \geqslant \bar{t}-\gamma_{1,-}^{-1}(x ; 0, \bar{t}) \\
& \geqslant c_{1} x^{1 / p} \quad \text { if } \bar{t} \geqslant t \geqslant \gamma_{1,-}^{-1}(x ; 0, \bar{t}) .
\end{aligned}
$$

The case $k=n_{1}+2$ follows immediately from (2.5)(ii) and (4.7), (4.8). Let us suppose now that hypothesis (4.6) is true for $k$. Then

$$
\begin{aligned}
\left(\partial_{x}^{k} u_{2}\right)(x, t)= & \int_{0}^{t}\left(\partial_{x}^{k} f_{2}\right)(x, q) d q+\partial_{x}^{k} u^{0}(x) \\
& +\sum_{s=0}^{k-\left(n_{1}+1\right)-1} \partial_{x}^{k-\left(n_{1}+1\right)-s-1}\left[\left(\partial_{x}^{s+n_{1}+1} f_{2}(\mathbf{u})\right) \partial_{x} \gamma_{1,-}^{-1}\right]_{\left\langle x, \gamma_{1,-}^{-1}(x ; 0, t)\right\rangle} .
\end{aligned}
$$

Using basically the same procedure of Lemma 3.1 we get

$$
\left.\begin{array}{rl}
\left(\partial_{x}^{k+1} u_{2}\right)(x, t)= & \int_{0}^{t}\left\{\sum_{i=1}^{2} \partial_{i} f_{2} \partial_{x}^{k+1} u_{i}+\sum_{\substack{\left.\sum_{i=1}^{n}\left|\alpha^{i}\right| \leqslant k+1 ; \alpha_{1}^{i}, \ldots, \alpha_{k+1}^{i}\right\} \\
i=1, \ldots, n}} \bar{h}_{\alpha}(\mathbf{u}) \prod_{j, i} \partial_{x}^{\alpha_{j}^{i}} u_{i}\right. \\
\left.+\sum_{i=3}^{n}\left(\lambda_{i}^{-1} \partial_{i} f_{2} X_{i} \partial_{x}^{k} u_{i}+\left(\partial_{t} \lambda_{i}\right) \partial_{i} f_{2} \partial_{x}^{k} u_{i}\right)\right\}(x, q) d q
\end{array}\right\}
$$


where $L_{k+1}$ is a smooth function of its arguments. We can rewrite (4.10) in the form

$$
\left(\partial_{x}^{k+1} u_{2}\right)(x, t)=\int_{0}^{t}\left(\sum_{i=1}^{2} \partial_{i} f_{2} \partial_{x}^{k+1} u_{i}\right)(x, q) d q+R(\mathbf{u}, x, t)
$$

An estimate for $R(\mathbf{u}, x, t)$ is easily derived using the inductive hypothesis and the expression for $\lambda_{1}\left(x, \gamma_{1,-}^{-1}(x ; 0, \bar{t})\right)$ :

$$
\lambda_{1}\left(x, \gamma_{1,-}^{-1}(x ; 0, \bar{t})\right)=x^{1-1 / p} f\left(x, \gamma_{1,-}^{-1}(x ; 0, \bar{t})\right)
$$

where $f$ is $C^{\infty}\left(R_{T}\right)$. We have

$$
|R(\mathbf{u}, x, t)| \leqslant c_{3} x^{-(k+1)+n_{1}+1+1 / p}, \quad(x, t) \in \Omega_{2}^{\beta_{1}} .
$$

In fact, the most singular term in $R(\mathbf{u}, x, t)$ is

$$
\left[\left(\partial_{x}^{k-\left(n_{1}+1\right)} \lambda_{1}^{-1}\right)\left(\partial_{x}^{n_{1}+1} f_{2}\right)\right]_{\left\langle x, \gamma_{1,-}^{-1}(x ; 0, \bar{t})\right\rangle},
$$

which satisfies the desired bound. To bound the derivative of $u_{1}$ we can now use the usual procedure:

$$
X_{1} \partial_{x}^{k} u_{1}=\left(\partial_{1} f_{1}+c_{k} \partial_{x} \lambda_{1}\right) \partial_{x}^{k} u_{1}+\sum_{i=2}^{n} \partial_{i} f_{1} \partial_{x}^{k} u_{i}+F_{1, k-1}(\mathbf{u}, x, t)
$$

where

$$
F_{1, k-1}=\sum_{\alpha^{i}, \sum_{i}\left|\alpha^{i}\right| \leqslant k ; \alpha_{j}^{i} \leqslant k-1} h_{\alpha}(\mathbf{u}, x, t) \prod_{i, j} \partial_{x}^{\alpha_{j}^{i}} u_{i}
$$

Let

$$
M_{1}(x, t)=\int_{0}^{t}\left(\partial_{i} f_{1}+c_{k} \partial_{x} \lambda_{1}\right)\left(\gamma_{1}(r ; x, t), r\right) d r
$$

then

$$
\begin{array}{r}
\left(\partial_{x}^{k} u_{1}\right)(x, t)=e^{M_{1}(x, t)}\left\{\int_{0}^{t} e^{-M_{1}(x, r)} \sum_{i=2}^{n}\left(\partial_{i} f_{1} \partial_{x}^{k} u_{i}+F_{1, k-1}\right)\left(\gamma_{1}(r ; x, t), r\right) d r\right. \\
\left.+\left(\partial_{x}^{k} u^{0}\right)\left(\gamma_{1,-}^{-1}(0, x, t)\right)\right\} \\
\forall(x, t) \in \Omega_{2}^{\beta_{1}},
\end{array}
$$

and

$$
\left|\left(\partial_{x}^{k+1} u_{1}\right)(x, t)\right| \leqslant c_{1} \int_{0}^{t}\left|\partial_{2} f_{1} \partial_{x}^{k+1} u_{2}\left(\gamma_{1}(r ; x, t), r\right)\right| d r+R_{1}(\mathbf{u}, x, t),
$$

where

$$
\left|R_{1}(\mathbf{u}, x, t)\right| \leqslant c_{2} x^{-(k+1)+n_{1}+1+1 / p} .
$$


We can now substitute (4.15) inside (4.11). Let $t_{\varepsilon}$ be a positive number to be chosen later; we obtain

$$
\begin{aligned}
& \sup _{\substack{(x, t) \in \Omega_{2}^{\beta_{1}} \\
t \leqslant t_{\varepsilon}}}\left|x^{k+1-\left(n_{1}+1+1 / p\right)} \partial_{x}^{k+1} u_{2}(x, t)\right| \\
& \leqslant \int_{0}^{t_{\varepsilon}} \sup _{\substack{(x, t) \in \Omega_{2}^{\beta_{1}} \\
t \leqslant t_{\varepsilon}}}\left|x^{k+1-\left(n_{1}+1+1 / p\right)} \partial_{x}^{k+1} u_{2}(x, t)\right|\left|\partial_{2} f_{2}(x, r)\right| d r+\left(\sup \left|\partial_{1} f_{2}\right|\right) \\
& \quad \sup _{(x, t) \in \Omega_{2}^{\beta_{1}}}\left\{x^{k+1-\left(n_{1}+1+1 / p\right)} \int_{0}^{t} d r \int_{0}^{r} d s\left|\partial_{2} f_{1} \partial_{x}^{k+1} u_{2}\left(\gamma_{1}(s ; x, r), s\right)\right|+c_{1}\right\} \\
& \leqslant c_{2}+\sup _{\substack{(x, t) \in \Omega_{2}^{\beta_{1}} \\
t \leqslant t_{\varepsilon}}}\left|x^{k+1-\left(n_{1}+1+1 / p\right)} \partial_{x}^{k+1} u_{2}\right| t_{\varepsilon} \sup \left|\partial_{2} f_{2}\right| \\
& +\left(\sup \left|\partial_{2} f_{1}\right| \sup \left|\partial_{1} f_{2}\right|\right) \sup _{\substack{(x, t) \in \Omega_{2}^{\beta_{1}} \\
t \leqslant t_{\varepsilon}}}\left[\int_{0}^{t} d r \int_{0}^{r} d s\left(\frac{x}{\gamma_{1}(s ; x, r)}\right)^{k+1-\left(n_{1}+1+1 / p\right)}\right.
\end{aligned}
$$

Recalling (2.7) and (4.7), we have the desired estimate for $\partial_{x}^{k+1} u_{2}$, provided that $t_{\varepsilon}$ is small enough. One can iterate this argument sufficiently many times in order to get the estimate in all of $\Omega_{2}^{\beta_{1}}$. In fact, the time interval $t_{\varepsilon}$ depends only on $f_{1}, f_{2}$, and the geometry of the system. Using this estimate for $\partial_{x}^{k+1} u_{2}$ and (4.15), one can easily obtain the estimate for $\partial_{x}^{k+1} u_{1}$ (actually better because the absence of the term created by the jump discontinuity across $C_{1}$ in $\partial_{x}^{k+1} u_{2}$ lets us gain a factor $x^{1 / p}$ ).

We can now go on to the estimate for $X_{1}^{s_{1}} \partial_{x}^{s_{2}} u_{i}, i=1,2$, and $n_{1}+1 \leqslant s_{1}+s_{2} \leqslant k$ +1 . If $i=1$ and $s_{1} \geqslant 1$, we can change the order of the derivatives and use the system and the inductive hypothesis to get the desired result. We focus on the case $X_{1}^{s_{1}} \partial_{x}^{s_{2}} u_{2}$.

We start with the region $\Omega_{2}^{\beta_{1}}$, where we already know the bound on $\partial_{x}^{k+1} u_{i}$, $i=1,2$. We can express $X_{1}^{s_{1}} \partial_{x}^{s_{2}} u_{2}$ in terms of $\partial_{x}^{s_{1}+s_{2}} u_{2}$ and $X_{1}^{\alpha} \partial_{x}^{\beta} u_{j}, \alpha+\beta<s_{1}+s_{2}$ :

$$
\begin{aligned}
& X_{1}^{s_{1}} \partial_{x}^{s_{2}} u_{2}=\sum_{\alpha^{i}, \beta^{i}, i=1, \ldots, n} h_{\alpha, \beta}(\mathbf{u})(t-\bar{t})^{l\left(\sum_{i}\left|\beta^{i}\right|-s_{2}, s_{1}-\sum_{i}\left|\alpha^{i}\right|\right)} \\
& \sum_{i}\left(\left|\alpha^{i}\right|+\left|\beta^{i}\right|\right) \leqslant s_{1}+s_{2}-1 \\
& \Sigma_{i}\left|\alpha^{i}\right| \leqslant s_{1}-1 \\
& \cdot \prod_{i, j} X_{1}^{\alpha_{j}^{i}} \partial_{x}^{\beta_{j}^{i}} u_{i}+\alpha^{s_{1}}(t-\bar{t})^{s_{1}(p-1)} \partial_{x}^{s_{1}+s_{2}} u_{2},
\end{aligned}
$$

where $l(m, n)$ is given by (4.3). (The formula is easily proven by induction.) To get the desired estimates it is sufficient to plug in equality (4.17), the bound on $\partial_{x}^{s_{1}+s_{2}} u_{2}$, and the inductive hypothesis (4.6). For example, consider the last term in (4.17) (that is actually the most singular one). Using the bounds on $\partial_{x}^{s_{1}+s_{2}} u_{2}$ we get

$$
\begin{aligned}
\left|\alpha^{s_{1}}(t-\bar{t})^{s_{1}(p-1)} \partial_{x}^{s_{1}+s_{2}} u_{2}\right| & \leqslant c|t-\bar{t}|^{s_{1}(p-1)} x^{-\left(s_{1}+s_{2}\right)+\left(n_{1}+1\right)+1 / p} \\
& \leqslant x^{-s_{2}-s_{1 / p}+n_{2}+1+1 / p} \text { as in } \Omega_{2}^{\beta_{1}}|t-\bar{t}|^{p} \leqslant c_{1} x .
\end{aligned}
$$


We are now going to find a similar bound in the region $\Omega_{3}^{\beta_{1}}=\{(x, t): x>0$, $\left.t_{\beta_{1}}(x) \leqslant t \leqslant \gamma_{\beta}^{-1}(x)\right\}$ (see Figure 4.1). In this region we have, in the inductive hypothesis, the restriction $s_{2} \leqslant n+1$. The basic fact is that in this region the following relation between $x$ and $t$ holds:

$$
b_{1} x \geqslant(t-\bar{t})^{p} \geqslant b_{2} x .
$$

The technique is always the same, and we refer to $\S 3$ for further details. We already know from Theorem 3.1 that for $s_{2} \leqslant n+1$ the function $X_{1}^{s_{1}} \partial_{x}^{s_{2}} u_{2}$ is continuous in $\Omega_{3}^{\beta_{1}}$. Using (3.3) and (3.4) with $i=1, j=2$, and taking $\left(x, t_{1}\right)$ as initial point, where $t_{1}$ is a fixed number such that $t_{\beta_{1}}(x) \geqslant t_{1} \geqslant t_{\delta}(x), \delta<\beta_{1}\left(\left(x, t_{1}\right) \in \Omega_{2}^{\beta_{1}}\right)$, we have (4.19)

$$
\begin{aligned}
\left(X_{1}^{s_{1}-1} \partial_{x}^{s_{2}} u_{2}\right)(x, t)= & e^{M_{2}(x, t)}\left(X_{1}^{s_{1}-1} \partial_{x}^{s_{2}} u_{2}\right)\left(x, t_{1}\right) \\
& +\int_{t_{1}}^{t} e^{M_{2}(x, t)-M_{2}(x, q)}\left(\sum_{\substack{i=1 \\
i \neq 2}}^{n} \partial_{i} f_{2} X_{1}^{s_{1}-1} \partial_{x}^{s_{2}} u_{i}+F_{2, s_{1}-2}\right)(x, q) d q,
\end{aligned}
$$

where we have the following expressions for $M_{2}$ and $F_{2, s_{1}-2}$ :

$$
\begin{aligned}
\exp \left(M_{2}(x, t)-M_{2}(x, r)\right) \\
=\exp \left(\int_{r}^{t}\left[\partial_{2} f_{2}+X_{1} \log \frac{\lambda_{1}}{\partial_{2} \gamma_{1}}\right](x, q) d q\right) \\
\quad+\exp \left(\int_{r}^{t} \partial_{2} f_{2}+\partial_{t}\left(\log \lambda_{1}\right)+\lambda_{1} \partial_{x} \log \lambda_{1}-X_{1} \log \partial_{2} \gamma_{1}(x, q) d q\right) \\
=\lambda_{1}(x, t) \lambda_{1}^{-1}(x, r) \exp \left(\int_{r}^{t} h(x, q) d q\right)=\lambda_{1}(x, t) \lambda_{1}^{-1}(x, r) e^{\tilde{M}_{2}(x, r, t)} .
\end{aligned}
$$

(ii)

$$
\begin{aligned}
& F_{2, s_{1}-2}=\sum_{\alpha^{i}, \beta^{i}} \quad f_{\alpha, \beta}(\mathbf{u}, x, t) \prod_{i, j} X_{1}^{\alpha^{i}} \partial_{x}^{\beta^{i}} u_{i} \\
& \sum_{i}\left(\left|\alpha^{i}\right|+\left|\beta^{i}\right|\right) \leqslant s_{1}+s_{2}-2 \\
& \alpha_{j}^{i} \leqslant s_{1}-1, \beta_{j}^{i}-s_{2} \\
& +\sum_{r=1}^{s_{1}-2} \sum_{q=0}^{s_{1}-2-r}\left(X_{1}^{r+1} \frac{\lambda_{1}}{\partial_{s} \gamma_{1}}\right) \\
& \left\{\begin{array}{r}
C_{r, q}\left(X_{1}^{s_{1}-2-r-q} \partial_{2} \gamma_{1} \lambda_{1}^{-1}\right)\left(X_{1}^{q}\left(X_{1}-\partial_{t}\right) \partial_{x}^{s_{2}} u_{2}\right. \\
+\left(X_{1} \frac{\lambda_{1}}{\partial_{2} \gamma_{1}}\right) \partial_{1} \gamma_{1} \lambda_{1}^{-1} X_{1}^{s_{1}-2} \partial_{x}^{s_{2}} f_{2} \text { if } s_{2}=n_{1}+1, \\
C_{r, q}\left(X_{1}^{s_{1}-2-r-q} \partial_{2} \gamma_{1}\right) X_{1}^{q} \partial_{x}^{s_{2}+1} u_{2} \\
+\left(X_{1} \frac{\lambda_{1}}{\partial_{2} \gamma_{1}}\right)\left\{\sum_{q=1}^{s_{1}-2}\left(X_{1}^{q} \frac{\partial_{2} \gamma_{1}}{\lambda_{1}}\right) X_{1}^{s_{1}-2-q}\left(X_{1}-\partial_{t}\right) \partial_{x}^{s_{2}} u_{2}\right. \\
\left.+\frac{\partial_{2} \gamma_{1}}{\lambda_{1}} X_{1}^{s_{1}-2} \partial_{x}^{s_{2}} f_{2}\right\} \\
\text { if } s_{2}<n+1,
\end{array}\right.
\end{aligned}
$$

where $F_{\alpha, \beta}$ are smooth functions of their arguments. 
With the usual technique we compute $X_{1}^{s_{1}} \partial_{x}^{s_{2}} u_{2}$ starting from (4.19):

$\left(X_{1}^{s_{1}} \partial_{x}^{s_{2}} u_{2}\right)(x, t)$

$$
\begin{gathered}
=\left(X_{1} M_{2}(x, t)\right)\left(X_{1}^{s_{1}-1} \partial_{x}^{s_{2}} u_{2}\right)(x, t)+e^{M_{2}(x, t)} X_{1}\left(X_{1}^{s_{1}-1} \partial_{x}^{s_{2}} u_{2}\right)\left(x, t_{1}\right) \\
+\lambda_{1} e^{M_{2}(x, t)} \int_{t_{1}}^{t}\left\{\frac{1}{\lambda_{1}} X_{1}\left(e^{-M_{2}} F_{2, s_{1}-2}\right)+\left(\partial_{t} \lambda_{1}\right) F_{2, s_{1}-s} e^{-M_{2}}\right. \\
+\sum_{\substack{i=1 \\
i \neq 2}}^{n}\left[\frac{1}{\lambda_{i}} X_{i}\left(\partial_{i} f_{2} e^{-M_{2}} X_{1}^{s_{1}-1} \partial_{x}^{s_{2}} u_{i}\right)\right. \\
\left.\left.\times\left(\partial_{t} \lambda_{i}\right) \partial_{i} f_{2} e^{-M_{2}} X_{1}^{s_{1}-1} \partial_{x}^{s_{2}} u_{i}\right]\right\}(x, r) d r \\
+e^{M_{2}} \frac{\lambda_{1}(x, t)}{\lambda_{1}\left(x, t_{1}\right)}\left(F_{2, s_{1}-2} e^{-M_{2}}+e^{-M_{2}} \partial_{1} f_{2} X_{1}^{s_{1}-1} \partial_{x}^{s_{2}} u_{1}\right)\left(x, t_{1}\right) \\
+e^{M_{2}} \lambda_{1}(x, t) \sum_{i>2}^{n}\left\{\left(\frac{e^{-M_{2}}}{\lambda_{i}} \partial_{i} f_{2} X_{1}^{s_{1}-1} \partial_{x}^{s_{2}} u_{i}\right)\left(x, t_{1}\right)\right. \\
\left.+\left[e^{-M_{2}}\left(\lambda_{i}^{-1}-\lambda_{1}^{-1}\right) \partial_{i} f_{2} X_{1}^{s_{1}-1} \partial_{x}^{s_{2}} u_{i}\right]\left\langle x, \gamma_{1}^{-1},(x ; 0, \tilde{t})\right\rangle\right\}
\end{gathered}
$$

We know how to bound all the derivatives appearing on the r.h.s. of (4.21). In fact, after having applied the system, the only derivative of order $k+1$ present in the formula is

$$
X_{1}\left(\left(X_{1}^{s_{1}-1} \partial_{x}^{s_{2}} u_{2}\right)\left(x, t_{1}\right)\right)=\left(\lambda_{1} \partial_{x} X_{1}^{s_{1}-1} \partial_{x}^{s_{2}} u_{2}\right)\left(x, t_{1}\right)
$$

for which we can first change the order of the derivatives and then apply the estimates valid in region $\Omega_{2}^{\beta_{1}}$. The most singular term in (4.21) is

$$
e^{M_{2}(x, t)} \lambda_{1}(x, t) \int \frac{1}{\lambda_{1}} X_{1}\left(e^{-M_{2}}\left(F_{2, s_{1}-2}+\partial_{1} f_{2} X_{1}^{s_{1}-1} \partial_{x}^{s_{2}} u_{1}\right)\right)(x, r) d r .
$$

We show how to deal with it. The remaining terms can be treated likewise and turn out to be much easier.

Recalling (4.18) (still valid if $\beta_{1}$ is replaced by $\delta$ ) and (4.20)(ii), we have the following bound for the integrand:

$$
\begin{aligned}
& \left|\left\{\lambda_{1}(x, t) e^{M_{2}(x, t)} \lambda_{1}^{-1} X_{1}\left[e^{-M_{2}}\left(\partial_{1} f X_{1}^{s_{1}-1} \partial_{x}^{s_{2}} u_{1}+F_{2, s_{1}-2}\right)\right]\right\}(x, r)\right| \\
& \leqslant \mid c_{1} \frac{\lambda_{1}^{2}(x, t)}{\lambda_{1}(x, r)}\left[\frac{e^{\tilde{M}_{2}}}{(r-\bar{t})^{p}}\left(\partial_{1} f_{2} X_{1}^{s_{1}-1} \partial_{x}^{s_{2}} u_{1}+F_{2, s_{1}-2}\right) \mid\right. \\
& \left.\quad+\frac{e^{\tilde{M}_{2}}}{(r-\bar{t})^{p-1}} X_{1}\left(\partial_{1} f_{2} X_{1}^{s_{1}-1} \partial_{x}^{s_{2}} u_{1}+F_{2, s_{1}-1}\right)\right] \mid(x, r) \\
& \leqslant c_{2} \frac{\lambda_{1}(x, t)}{(r-\bar{t})^{p-1}}\left[\frac{1}{(r-\bar{t})}\left(x^{-s_{1} / p-s_{2}+n_{1}+1+2 / p}+1\right)\right. \\
& \left.+\left(1+x^{-s_{1} / p-2_{2}+n_{1}+1+1 / p}\right)\right] .
\end{aligned}
$$


Hence,

$$
\begin{aligned}
& \left|X_{1}^{s_{1}} \partial_{x}^{s_{2}} u_{2}(x, t)\right| \\
& \quad \leqslant c_{1} \int_{t_{1}}^{t} \frac{\lambda_{1}(x, t)}{(r-\bar{t})^{p-1}}\left[\frac{1}{(r-\bar{t})}\left(x^{-s_{1} / p-s_{2}+n_{1}+1+2 / p}\right)+x^{-s_{1} / p-s_{2}+n_{1}+1+1 / p}+1\right] d r \\
& \quad \leqslant c_{2} x^{-s_{1} / p-s_{2}+n_{1}+1+1 / p} .
\end{aligned}
$$

This completes the proof of Step 2.

Step 3. Continuity of $X_{r}^{k+1}\left(\partial_{t} X_{1}\right)^{n_{1}+1} u_{i}, r=1,2, i=1, \ldots, n$, across $C_{j}, j \geqslant 3$, for $k+1 \leqslant\left(n_{1}+n_{1}+1+2\right) p-2\left(n_{1}+1\right)$.

First of all we reduce the problem to proving the continuity of $X_{r}^{k+1}\left(\partial_{t} X_{1}\right)^{n_{1}+1} u_{j}$ across $C_{j}, j \geqslant 3$. In fact, if we assume that our inductive hypotheses are true for $k$, and we consider $u_{i}$ near $C_{j}$ for $i \neq j$, we have that $X_{i} X_{1}^{k}\left(\partial_{t} X_{1}\right)^{n_{1}+1} u_{i}$ and $X_{j} X_{1}^{k}\left(\partial_{t} X_{1}\right)^{n_{1}+1} u_{i}$ are continuous together with $X_{j} X_{1}^{k}\left(\partial_{t} X_{1}\right)^{n_{1}+1} u_{j}$ (the first using the system, the latter using a technique like the one in $\S 3$ ). The only other derivative we need to examine in order to show that the solution

$$
\mathbf{u} \in C^{2\left(n_{1}+1\right)+k+1}\left(R_{T} \backslash\left(C_{1} \cup C_{2}\right)\right)
$$

is either $X_{1}^{k+1}\left(\partial_{t} X_{1}\right)^{n_{1}+1} u_{j}$ or $\partial_{t}^{k+1}\left(\partial_{t} X_{1}\right)^{n_{1}+1} u_{j}, j \geqslant 3$. We choose the first or the second according to the sign of $\lambda_{j}$. We fix the index $j$ from now on. Let us assume for definiteness that $\lambda_{j}$ is positive; we then choose to show the continuity of $X_{1}^{k+1}\left(\partial_{t} X_{1}\right)^{n_{1}+1} u_{j}$ across $C_{j}$. We first need a change of coordinates that symmetrizes the vector fields $X_{1}$ and $\partial_{t}$ and, at the same time, makes the vector field $X_{j}$ parallel to $\partial_{x}$.

Let $\phi$ be the function $\phi:(x, t) \rightarrow\left(x, y_{j}\right), y_{j}=\gamma_{j}^{-1}(0 ; x, t)$, and let $\psi$ be the function $\psi:\left(x, y_{j}\right) \rightarrow\left(w, y_{j}\right), w=\gamma\left(\bar{t} ; x, y_{j}\right)$, where $\gamma\left(r ; x, y_{j}\right)$ is the curve through $\left(x, y_{j}\right)$ such that

$$
\frac{\partial}{\partial r} \gamma\left(r ; x, y_{j}\right)=\left(\frac{\lambda_{1}}{2 \eta}\right)\left(\gamma\left(r ; x, y_{j}\right), r\right) \text {. }
$$

We have that

$$
\begin{gathered}
X_{1} \stackrel{\psi \circ \phi}{\rightarrow} \eta X_{+}=\eta\left(\begin{array}{c}
\tilde{\lambda}_{1} \\
1
\end{array}\right), \quad \partial_{t} \stackrel{\psi \circ \phi}{\rightarrow} \mathcal{O} X_{-}=\mathcal{O}\left(\begin{array}{c}
-\tilde{\lambda}_{1} \\
1
\end{array}\right), \\
X_{j} \stackrel{\psi \circ \phi}{\rightarrow} \tilde{X}_{j}=\frac{\lambda_{j}}{\partial_{2} \gamma}\left(\begin{array}{c}
-1 \\
0
\end{array}\right)=\tilde{\lambda}_{j}\left(\begin{array}{l}
1 \\
0
\end{array}\right),
\end{gathered}
$$

where $\eta, \mathcal{O}, \tilde{\lambda}_{j}$ are smooth functions bounded away from zero in $R_{T}$. $C_{1}$ is mapped onto $\gamma_{+}\left(y_{j} ; 0, \bar{t}\right)$ and $C_{2}$ onto $\gamma_{-}\left(y_{j} ; 0, \bar{t}\right)$, symmetric curves with respect to the $y_{j}$ axis. 
REMARK 4.2. The line $w=0$ in the $\left(w, y_{j}\right)$-plane is mapped by $\phi^{-1} \circ \psi^{-1}$ onto the curve through the point $(0, \bar{t})$ tangent to the vector field $\left(\lambda_{1} / 2 z, 1\right)$ in the plane $(x, t)$, with $z=1-\lambda_{1} / 2 \lambda_{j}$.

We can now start to compute higher order derivatives using (4.4). We apply the following rules:

(4.24) (a) On the term containing $(t-\bar{t})^{2\left(n_{1}+1\right)(p-1)} \partial_{x}^{n_{1}+1} u_{1} \partial_{x}^{n_{1}+1} u_{2}$ we take the right kind of derivative near each $C_{i}, i=1,2$, so that the integral makes sense. Figure 4.2 describes the procedure:

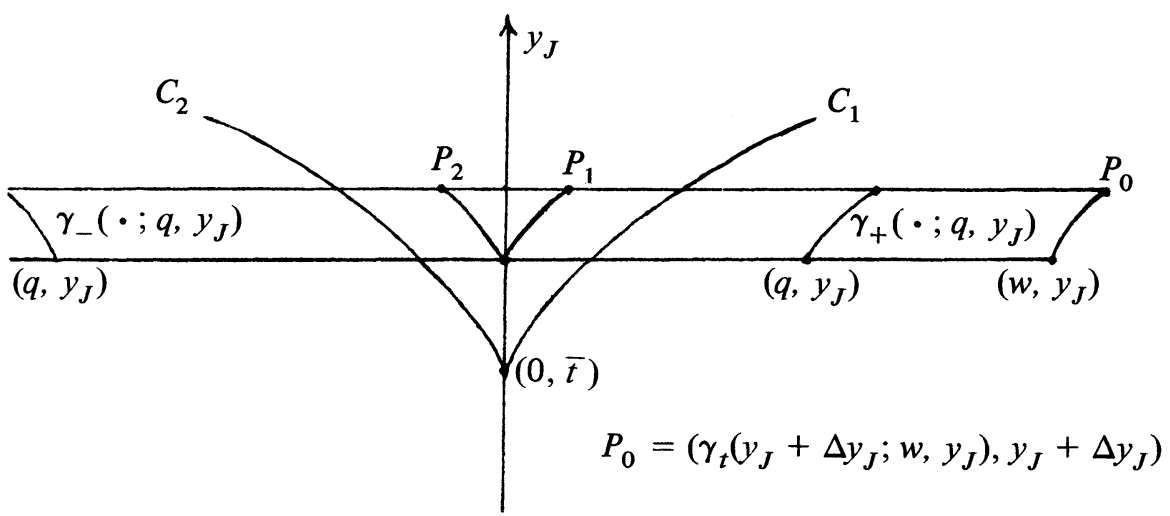

\section{FIGURE 4.2}

The picture describes the paths of the two integrals in the difference quotient and shows how to compare the two integrands along the right characteristics. The segment $\overline{P_{1} P_{2}}$ gives a term at $\left(0, y_{j}\right)$. Namely, working in the $\left(w, y_{j}\right)$-plane we have

$$
\begin{aligned}
X_{+} \int_{w_{0}}^{w} F\left(\mathbf{u} ; q, y_{j}\right) d q= & \left(-\lambda_{1} F\right)\left(w_{0}, y_{j}\right)+\int_{w_{0}}^{0}\left\{X_{-} F+\left(-\partial_{w} \tilde{\lambda}_{1}\right) F\right\}\left(q, y_{j}\right) d q \\
& +\int_{0}^{w}\left\{X_{+} F+\left(\partial_{w} \tilde{\lambda}_{1}\right) F\right\}\left(q, y_{j}\right) d q+\left(2 \tilde{\lambda}_{1} F\right)\left(0, y_{j}\right) .
\end{aligned}
$$

(b) Inside the integrals containing $(t-\bar{t})^{l\left(n_{1}+1+s, 2\left(n_{1}+1\right)\right)} \partial_{x}^{s} u_{1} \partial_{x}^{n_{1}+1} u_{2}, s \leqslant n_{1}$, we take a $\partial_{t}$ derivative. This also gives a term at $\left(w, y_{j}\right)$.

(c) The term $R_{1}$ in (4.4) is the sum of $n-3$ terms like $h_{i}\left(\partial_{t} X_{1}\right)^{n_{1}+1} u_{i}$; in front of each of them we create the operator $X_{i}$; this also gives $n-3$ terms at $\left(w, y_{j}\right)$.

(d) In front of the remaining terms we take an $X_{1}$ derivative.

(e) Since $M_{j}=\int_{w_{0}}^{w} h\left(\mathbf{u}, q, y_{j}\right) d q$, we compute its derivatives by first creating inside the integral the operator $L$ and then mimicking the procedure described in (a)-(d). Let $F_{+}, F_{-}, F_{i}$ be the differential operators defined by

$$
\left.\underset{(-)}{F_{+} f}=\underset{(-)}{X_{+} f+\left(\partial_{x}\right.} \tilde{\lambda}_{1}\right) f ; \quad F_{i} f=X_{i} f+\left(\partial_{w} \tilde{\lambda}_{i}\right) f .
$$


Applying rules (4.24) to formula (4.7) we get

$$
\begin{aligned}
& \left(X_{1}\left(\partial_{t} X_{1}\right)^{n_{1}+1} u_{j}\right)\left(x\left(w, y_{j}\right), t\left(w, y_{j}\right)\right) \\
& =\left(X_{1} M_{j}(x, t)\right)\left(\partial_{t} X_{1}\right)^{n_{1}+1} u_{j}\left(x\left(w, y_{j}\right), t\left(w, y_{j}\right)\right)+e^{M_{j}\left(x\left(w, y, y_{j}\right), t\left(w, y_{j}\right)\right)} \\
& \left\{i_{2\left(n_{1}+1\right)+1}\left(\mathbf{u}^{0}\right)\right. \\
& +\int_{w_{0}}^{0} F_{-}\left(e^{-M_{j}}(t-\bar{t})^{2\left(n_{1}+1\right)(p-1)} \tilde{L}^{n_{1}+1, n_{1}+1} \partial_{x}^{n_{1}+1} u_{1} \partial_{x}^{n_{1}+1} u_{2}\right)\left(x\left(q, y_{j}\right), t\left(q, y_{j}\right)\right) d q \\
& +\left(2 \tilde{\lambda}_{1} e^{-M_{j}} \tilde{L}^{n_{1}+1, n_{1}+1}(t-\bar{t})^{2\left(n_{1}+1\right)(p-1)} \partial_{x}^{n_{1}+1} u_{1} \partial_{x}^{n_{1}+1} u_{2}\right)\left(x\left(0, y_{j}\right), t\left(0, y_{j}\right)\right) \\
& +\int_{0}^{w} F_{+}\left(e^{-M_{j}}(t-\bar{t})^{2\left(n_{1}+1\right)(p-1)} \tilde{L}^{n_{1}+1, n_{1}+1} \partial_{x}^{n_{1}+1} u_{1} \partial_{x}^{n_{1}+1} u_{2}\right)\left(x\left(q, y_{j}\right), t\left(q, y_{j}\right)\right) d q \\
& +\int_{w_{0}}^{w_{1}} F_{+}\left(\sum_{\substack{r, s=s \\
s-n_{1}}}^{n_{1}+1} e^{-M_{j}}(t-\bar{t})^{\left(\left(r+s, 2\left(n_{1}+1\right)\right)\right.} \tilde{L}^{r \cdot s} \partial_{x}^{r} u_{1} \partial_{x}^{s} u_{2}\right)\left(x\left(q, y_{j}\right), t\left(q, y_{j}\right)\right) d q \\
& +\int_{u^{\prime} 0}^{u_{1}} F_{-}\left(\sum_{r=0}^{n_{1}} e^{-M_{j}}(t-\bar{t})^{l\left(r+n_{1}+1.2\left(n_{1}+1\right)\right)} \tilde{L}^{r \cdot n_{1}+1} \partial_{x}^{r} u_{1} \partial_{x}^{n_{1}+1} u_{2}\right)\left(x\left(q, y_{j}\right), t\left(q, y_{j}\right)\right) d q \\
& +\left(2 \tilde{\lambda}_{1} e^{-M_{j}} \sum_{r=0}^{n_{1}}(t-\tilde{t})^{\left(\left(r+n_{1}+1.2\left(n_{1}+1\right)\right)\right.} \tilde{L}^{r \cdot n_{1}+1} \partial_{x}^{r} u_{1} \partial_{x}^{n_{1}+1} u_{2}\right)\left(x\left(w, y_{j}\right), t\left(w, y_{j}\right)\right) \\
& +\sum_{\substack{i=3 \\
i \neq j}}^{n}\left[\int_{w_{0}}^{w} F_{i}\left(e^{-M_{j}} h_{i}(\mathbf{u})\left(\partial_{t} X_{1}\right)^{n_{1}+1} u_{i}\right)\left(x\left(q, y_{j}\right), t\left(q, y_{j}\right)\right) d q\right. \\
& \left.\left.+\left(e^{-M_{j} h_{i}}\left[\left(\partial_{t} X_{1}\right)^{n_{1}+1} u_{i}\right]\left(\tilde{\lambda}_{1}-\tilde{\lambda}_{j}\right)\right)\left(x\left(w, y_{j}\right), t\left(w, y_{j}\right)\right)\right]\right\},
\end{aligned}
$$

where $L_{2\left(n_{1}+1\right)+1}$ is smooth in its arguments and $w_{0}=w\left(\gamma_{j}^{-1}(0, x, t), 0\right)$. Expanding the derivative in the last integral and using the system, we get an expression like

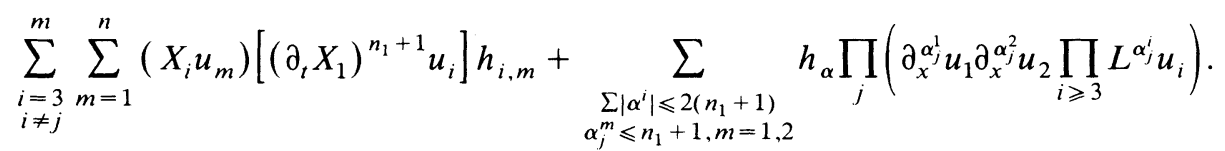

Using this expression it is easy to recognize that in the r.h.s. of (4.25) there are no derivatives of order $2\left(n_{1}+1\right)+1$ of $\mathbf{u}$ (unless $n_{1}=-1$, but this case will be treated separately in Remark 4.3). Moreover, all the functions contained in the integrands are bounded in the region of integration. 
REMARK 4.3. Our procedure is not valid if $n_{1}=-1$, since (4.4) does not hold in this case. To deal with this case we first notice that for $i=1,2$ the following formula holds (see 4.2):

$$
\begin{aligned}
\left(X_{i} j_{u}\right)(x, t)=e^{M_{j}(x, t)}\left[\left(X_{i} u_{j}\right)\left(x_{0}, t_{0}\right)\right. & \\
& \left.\quad+\int_{x_{0}}^{x} \frac{e^{-M_{j}}}{\lambda_{j}}\left(\sum_{\substack{r=1 \\
r \neq j}}^{n} \partial_{r} f_{j} X_{1} u_{r}+h_{0}(\mathbf{u})\left(q, \gamma_{j}^{-1}(q ; x, t)\right)\right) d q\right]
\end{aligned}
$$

Moreover,

$$
\left(X_{1} u_{j}\right)\left(x\left(0, y_{j}\right), t\left(0, y_{j}\right)\right)=\left[\left(1-\frac{\lambda_{1}}{\lambda_{j}}\right) \partial_{t} u_{j}+\frac{\lambda_{1}}{\lambda_{j}} f_{j}\right]\left(x\left(0, y_{j}\right), t\left(0, y_{j}\right)\right) .
$$

Applying twice the usual trick, we get

$$
\begin{aligned}
& \left(X_{1} y_{j}\right)\left(x\left(w, y_{j}\right), t\left(w, y_{j}\right)\right) \\
& =e^{M_{j}^{1}(x, t)}\left\{\left(X_{1} u_{j}\right)\left(x\left(0, y_{j}\right), t\left(0, y_{j}\right)\right)\right. \\
& \left.+\int_{0}^{w}\left[\frac{e^{-M_{j}^{1}}}{\lambda_{j}}\left(\sum_{\substack{r=1 \\
r \neq j}}^{n} \partial_{r} f_{j} X_{1} u_{r}+h_{0}\right)\right]\left(q, y_{j}\right) d q\right\} \\
& =e^{M_{j}^{1}(x, t)}\left\{\int_{0}^{w}\left[e^{-M_{j}^{1}}\left(\sum_{\substack{r=1 \\
r \neq J}}^{n} \partial_{r} f_{j} X_{1} u_{r}+h_{0}\right)\right](q, y) d q\right. \\
& +\left(\frac{\lambda_{1}}{\lambda_{j}} f_{j}\right)(x(0, y), t(0, y)) \\
& +\left(1-\frac{\lambda_{i}}{\lambda_{j}}\right) e^{M_{j}^{2}\left(0, y_{j}\right)}\left[L_{1}\left(u^{0}\left(\gamma_{j}^{-1}(0 ; x, \bar{t})\right)\right)\right. \\
& \left.\left.+\int_{w_{0}}^{0}\left[e^{-M_{j}^{2}}\left(\sum_{\substack{r=1 \\
r \neq J}} \partial_{r} f_{j} u_{r}+h_{0}\right)\right](q, y) d q\right\}\right] .
\end{aligned}
$$

Formula (4.26) is almost identical to the three terms in (4.25) corresponding to rule (4.24)(a). In fact, for the case $n_{1}=-1$, we can just keep applying that rule, avoiding the following complicated procedure. 
Examining the r.h.s. of (4.25) it is easy to get the continuity of $X_{1}\left(\partial_{t} X_{1}^{n_{1}+1} u_{j}\right)$ across $C_{j}$, as all the terms are either continuous across $C_{j}$ or (the ones at $\left.\left(0, y_{j}\right)\right)$ tend to zero as $(x, t)$ tends to $C_{j}$ (corresponding to $y_{j} \rightarrow \bar{t}$, which implies that $\tilde{\lambda}_{1}$ tends to zero). We can repeat the same argument for $j=3, \ldots, n$, obtaining that $\mathbf{u} \in$ $C^{2\left(n_{1}+1\right)+1}\left(R_{T} \backslash\left(C_{1} \cup C_{2}\right)\right)$.

For later purposes we rewrite (4.25) as

$$
\begin{aligned}
& \left(X_{1}\left(\partial_{t} X_{1}\right)^{n_{1}+1} u_{j}\right)(x, t) \\
& \quad=e^{M_{j}}\left(A_{j, a}^{1}+A_{j, b}^{1}+A_{j, d}^{1}+A_{j, p}^{1}\right)+\left(X_{1} M_{j}\right)\left(\partial_{t} X_{1}\right)^{n_{1}+1} u_{j},
\end{aligned}
$$

where

$$
\begin{aligned}
A_{j, p}^{i}= & \left(2 \tilde{\lambda}_{1} e^{-M_{j}} \sum_{r=0}^{n} \tilde{L}^{r, n_{1}+1} \partial_{x}^{r} u_{1} \partial_{x}^{n_{1}+1} u_{2}(t-\bar{t})^{\mu\left(r+n_{1}+1,2\left(n_{1}+1\right)\right)}\right)(x, t) \\
A_{j, u}^{1}= & \int_{w_{0}}^{0} F_{-}\left[\left(e^{-M_{j}}(t-\bar{t})^{2\left(n_{1}+1\right)(p-1)} \tilde{L}^{n_{1}+1, n_{1}+1} \partial_{x}^{n_{1}+1} u_{1} \partial_{x}^{n_{1}+1} u_{2}\right)\right]\left(x\left(q, y_{j}\right), t\left(q \cdot y_{j}\right)\right) d q \\
& +\int_{0}^{w} F_{+}\left(e^{-M_{j}}(t-\bar{t})^{2\left(n_{1}+1\right)(p-1)} \tilde{L}^{n_{1}+1, n_{1}+1} \partial_{x}^{n_{1}+1} u_{1} \partial_{x}^{n_{1}+1} u_{2}\right)(x(q, y), t(q, y)) d q \\
& +Z+\left(2 \tilde{\lambda}_{1} e^{-M_{j}} \tilde{L}^{n_{1}+1, n_{1}+1}(t-\bar{t})^{2\left(n_{1}+1\right)(p-1)} \partial_{x}^{n_{1}+1} u_{1} \partial_{x}^{n_{1}+2} u_{2}\right)\left(x\left(0, y_{j}\right), t\left(0, y_{j}\right)\right)
\end{aligned}
$$

$Z$ includes all the terms containing the factor $(t-\bar{t})^{2\left(n_{1}+1\right)(p-1)}$ created in the other integrals. In $A_{j, b}^{1}$ we gather the terms containing $\partial_{x}^{n_{1}+1} u_{2}$; in $A_{j, d}^{1}$, all the rest. The subscripts $a, b, d$ of $A_{j}^{1}$ refer to which of the rules (4.24) we are going to apply in taking the next derivative.

We now suppose that $\mathbf{u} \in C^{2\left(n_{1}+1\right)+k}\left(R_{T} \backslash\left(C_{1} \cup C_{2}\right)\right)$. We need an explicit expression for $X_{1}^{k}\left(\partial_{t} X_{1}\right)^{n_{1}+1} u_{j}$. We have it already for $k=1$ (4.25), and the following formula gives it for $k \leqslant 2\left(n_{1}+1\right)(p-1)+p$. One can then use rules (4.24) to compute $X_{1}^{k+1}\left(\partial_{t} X_{1}\right)^{n_{1}+1} u_{j}$, obtaining at the same time the continuity across $C_{j}$ and the proof of the formula itself by induction.

$$
\begin{aligned}
\left(X_{1}^{k}\left(\partial_{t} X_{1}\right)^{n_{1}+1} u_{j}\right)(x, t) & \\
=\sum_{l=0}^{k}\left(\begin{array}{c}
k \\
l
\end{array}\right)\left(X_{1}^{k-l} e^{M_{j}}\right)\{ & I_{2\left(n_{1}+1\right)+l}\left(\mathbf{u}^{0}\right) \\
& \left.+A_{j, a}^{l}(x, t)+A_{j, b}^{l}(x, t)+A_{j, d}^{l}(x, t)+A_{j, p}^{l}(x, t)\right\} .
\end{aligned}
$$

The expressions of $A_{j}^{l}$ are rather complicated, and before we can give them we need some notation.

Let $\alpha^{i}, \beta^{i}$ be $2 n$ multi-indices. Then we define

$$
\begin{gathered}
\text { (i) } H_{\alpha, \beta}^{s}(\mathbf{u})=\prod_{r}\left(X_{s}^{\beta_{r}^{1}} \partial_{x}^{\alpha_{r}^{1}} u_{1}\right)\left(X_{s}^{\beta_{r}^{2}} \partial_{x}^{\alpha_{r}^{2}} u_{2}\right) \prod_{i}\left(X_{s}^{\beta_{r}^{i}} L^{\alpha_{r}^{i}} u_{i}\right), \quad s=1,2, \\
\text { (ii) } W_{\alpha \beta}(\mathbf{u})=\prod_{i=3, \ldots, \ldots, n} A_{i, a}^{\alpha_{r}^{i}} A_{i, b}^{\alpha_{r}^{i}} A_{i, d}^{\alpha_{r}^{i}} B_{i, a}^{\beta_{r}^{i}} B_{i, b}^{\beta_{r}^{i}} B_{i, d}^{\beta_{r}^{i}} .
\end{gathered}
$$

The $B_{j}^{s}$ 's are defined in (4.30), which gives a compact expression for $X_{1}^{l} M_{j}$. The procedure used to compute this derivative is similar to rules (4.24), and we write, similarly to (4.27) (4.28),

$$
X_{1}^{s} M_{j}=B_{j, a}^{s}+B_{j, b}^{s}+B_{j, d}^{s}+B_{j, p}^{s} .
$$


If $s \leqslant 2\left(n_{1}+1\right)$ then the $B_{j}^{s}$ 's are given by

(4.31)

$$
B_{j, a}^{s}=B_{j, b}^{s}=0
$$

$$
B_{j, p}^{s}(x, t)=\sum_{\substack{\alpha^{i}=\left\{\alpha_{1}^{i}, \ldots, \alpha_{s}^{i}\right\}, i=1, \ldots, n \\ \sum_{i}\left(\left|\alpha^{1}\right|+\left|\beta^{i}\right|\right) \leqslant s-1 \\ \alpha_{r}^{1}, \alpha_{r}^{2} \leqslant n_{1}+1}} h_{\alpha}(\mathbf{u}, x, t)(t-\bar{t})^{l\left(\left|\alpha^{1}\right|+\left|\alpha^{2}\right|, s\right)+p-1} H_{\alpha, \beta} ;
$$

$$
B_{j, d}^{s}(x, t)=\int_{w_{0}}^{w(x, t)} \sum_{\substack{\alpha^{i} \\ \sum_{i}\left|\alpha^{i}\right| \leqslant s \\ \alpha_{r}^{1}, \alpha_{r}^{2} \leqslant[(s+1) / 2]}}\left(h_{\alpha}(\mathbf{u}, x, t)(t-\bar{t})^{l\left(\left|\alpha^{1}\right|+\left|\alpha^{2}\right|, s\right)} H_{\alpha, 0}\right)
$$

$$
\cdot\left(x\left(q, y_{j}\right), t\left(q, y_{j}\right)\right) d q .
$$

If $s>2\left(n_{1}+1\right)$ we define (recalling that $y_{j}=y_{j}(x, t), w=w(x, t)$ )

$$
\begin{aligned}
& B_{j, a}^{s}(x, t)=\int_{0}^{w(x, t)}\left[\sum_{k=0}^{s-2\left(n_{1}+1\right)}(t-\bar{t})^{l\left(2\left(n_{1}+1\right), 2\left(n_{1}+1\right)+k\right)}\right. \\
& \text {. } \left.\sum_{\substack{\alpha^{i}, \beta^{i} \\
\sum_{i}\left(\left|\alpha^{i}\right|+\left|\beta^{1}\right|\right) \leqslant s-k \\
\max _{i \geqslant 3, r}\left(\alpha_{r}^{1}, \alpha_{r}^{2}, \alpha_{r}^{i} / 2\right) \leqslant n_{1}+1}} h_{\alpha, \beta} H_{\alpha, \beta}^{1}\right]\left(x\left(q, y_{j}\right), t\left(q, y_{j}\right)\right) d q \\
& +\int_{w_{0}(x, t)}^{0}\left[\sum_{k=0}^{s-2\left(n_{1}+1\right)}(t-\bar{t})^{l\left(2\left(n_{1}+1\right), 2\left(n_{1}+1\right)+k\right)}\right. \\
& \begin{array}{c}
\cdot \sum_{\substack{\alpha^{i}, \beta^{i} \\
\sum_{i}\left(\left|\alpha^{i}\right|+\left|\beta^{i}\right|\right) \leqslant s-k \\
\max _{i \geqslant 3, r}\left(\alpha_{r}^{1}, \alpha_{r}^{2}, \alpha_{r}^{i} / 2\right) \leqslant n_{1}+1}} h_{\alpha, \beta}^{2} H_{\alpha, \beta}^{2} \\
+\sum_{r=0}^{s-2\left(n_{1}+1\right)}\left\{X_{1}^{\left[s-2\left(n_{1}+1\right)-1-r\right]} \sum_{k=0}^{r}(t-\bar{t})^{l\left(2\left(n_{1}+1\right), 2\left(n_{1}+1\right)+k\right)+p-1}\right.
\end{array}
\end{aligned}
$$$$
\left.\sum_{\substack{\alpha^{i}, \beta^{i} \\ \sum_{i}\left(\left|\alpha^{i}\right|+\left|\beta^{i}\right|\right) \leqslant 2\left(n_{1}+1\right)+r-k \\ \max _{i \geqslant 3, r}\left(\alpha_{r}^{1}, \alpha_{r}^{2}, \alpha_{r}^{i} / 2\right) \leqslant n_{1}+1}}^{s} h_{\alpha, \beta}^{s}\left(H_{\alpha, \beta}^{1}+H_{\alpha, \beta}^{2}\right)\right\}\left(x\left(0, y_{j}\right), t\left(0, y_{j}\right)\right) ;
$$ 


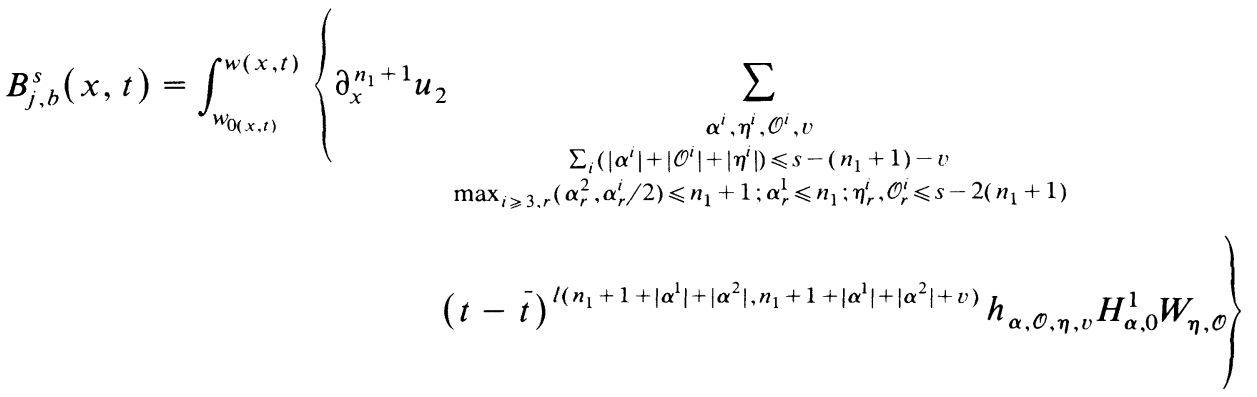

$$
\begin{aligned}
& \cdot(x(q, y), t(q, y)) d q \\
& B_{j, d}^{s}(x, t)=\int_{w_{0}(x, t)}^{w(x, t)} \sum_{\substack{\alpha^{i}, \eta^{i}, \mathcal{O}^{i}, v \\
\sum_{i}\left(\left|\alpha^{1}\right|+\left|\eta^{1}\right|+\mid \mathcal{O}^{i}\right) \leqslant s-v \\
\alpha_{r}^{2} \leqslant n_{1}: \alpha_{r}^{1}, \alpha_{r}^{i} / 2 \leqslant n_{1}+1 \\
\eta_{r}^{i}, \mathcal{O}_{r}^{i} \leqslant s-2\left(n_{1}+1\right)}}(t-\bar{t})^{l\left(\left|\alpha^{1}\right|+\left|\alpha^{2}\right|,\left|\alpha^{1}\right|+\left|\alpha^{2}\right|+v\right)} \\
& \cdot h_{\alpha, \eta, \mathcal{O}, v} H_{\alpha, 0} W_{\eta, \mathcal{O}}(x(q, y), t(q, y)) d q \text {; } \\
& \begin{aligned}
B_{j, p}^{s}(x, t)= & \sum_{\substack{\alpha^{i}, \beta^{i}, \eta^{i}, \mathcal{O}^{i}, v \\
\sum_{i}\left(\left|\alpha^{i}\right|+\left|\beta^{i}\right|+\left|\eta^{i}\right|+\mid \mathcal{O}^{1}\right) \leqslant s-v-1}}(t-\bar{t})^{l\left(\left|\alpha^{1}\right|+\left|\alpha^{2}\right|,\left|\alpha^{1}\right|+\left|\alpha^{2}\right|+v\right)+p-1} \\
& \max _{i \geqslant 3, r}\left(\alpha_{r}^{1}, \alpha_{r}^{2}, \alpha_{r}^{i} / 2\right) \leqslant n_{1}+1 ; \eta_{r}^{i}, \mathcal{O}_{r}^{i} \leqslant s-2\left(n_{1}+1\right) \\
& \cdot h_{\alpha, \eta, \mathcal{O}, \beta}^{v}\left(\mathbf{u}(x, t) H_{\alpha, \beta}^{1} W_{\eta}, \mathcal{O}\right)(x, t),
\end{aligned}
\end{aligned}
$$

where the functions $h$ depend smoothly on $\mathbf{u}, x\left(q, y_{j}(x, t)\right), t\left(q, y_{j}(x, t)\right), w(x, t)$, and $y_{j}(x, t)$.

The recursive definition (4.32) is consistent for $n_{1} \geqslant 0$, since it contains only $A_{i}^{r}$ and $B_{i}^{r}$ on the right side with $k \leqslant s-2\left(n_{1}+1\right)$.

We can now give the expressions for $A_{j}^{s}$, which are almost identical to the corresponding ones for $B_{j}^{s}$, except for $A_{j, p}^{s}$.

$$
A_{j, a}^{s}=\int_{0}^{w(x, t)}\left\{\sum_{k=0}^{s}(t-\bar{t})^{l\left(2\left(n_{1}+1\right), 2\left(n_{1}+1\right)+k\right)}\right.
$$

$$
\begin{gathered}
\sum_{\substack{\alpha^{i}, \beta^{i} \\
\sum_{i}\left(\left|\alpha^{i}\right|+\left|\beta^{i}\right|\right) \leqslant s-k+2\left(n_{1}+1\right) \\
\max _{i \geqslant 3, r}\left(\alpha_{r}^{1}, \alpha_{r}^{2}, \alpha_{r}^{i} / 2\right) \leqslant n_{1}+1}} g_{\alpha, \beta}(\mathbf{u}, x, t) e^{-M_{j}} H_{\alpha, \beta}^{1} \\
\cdot\left(x\left(q, y_{j}\right), t\left(q, y_{j}\right)\right) d q
\end{gathered}
$$




$$
\begin{aligned}
& +\int_{w_{0}}^{0}\left\{\sum_{r=0}^{s}(t-\bar{t})^{l\left(2\left(n_{1}+1\right), 2\left(n_{1}+1\right)+k\right)}\right. \\
& \left.\sum_{\substack{\alpha^{i}, \beta^{i} \\
\sum_{i}\left(\left|\alpha^{i}\right|+\left|\beta^{i}\right|\right) \leqslant s-k+2\left(n_{1}+1\right)}} e^{-M_{j} g_{\alpha, \beta}(\mathbf{u}, x, t) H_{\alpha, \beta}^{2}}\right\} \\
& \max _{i \geqslant 3, r}\left(\alpha_{r}^{1}, \alpha_{r}^{2}, \alpha_{r}^{i} / 2\right) \leqslant n_{1}+1 \\
& \cdot\left(x\left(q, y_{j}\right), t\left(q, y_{j}\right)\right) d q \\
& +\sum_{r=0}^{s-1} X_{1}^{s-1-r}\left\{\sum_{v=0}^{r}(t-\bar{t})^{l\left(2\left(n_{1}+1\right), 2\left(n_{1}+1\right)+v\right)+p-1}\right. \\
& \begin{array}{l}
\left.\sum_{\substack{\alpha^{i}, \beta^{i} \\
\sum_{i}\left(\left|\alpha^{i}\right|+\left|\beta^{i}\right|\right) \leqslant 2\left(n_{1}+1\right)+r-v \\
\max _{i \geqslant 3, r}\left(\alpha_{r}^{1}, \alpha_{r}^{2}, \alpha_{r}^{i} / 2\right) \leqslant n_{1}+1}} e^{-M_{j}} g_{\alpha, \beta}^{3}\left(H_{\alpha, \beta}^{1}+H_{\alpha, \beta}^{2}\right)\right\}\left(0, y_{j}\right) ;
\end{array} \\
& \begin{array}{l}
\left.\sum_{\substack{\alpha^{i}, \beta^{i} \\
\sum_{i}\left(\left|\alpha^{i}\right|+\left|\beta^{i}\right|\right) \leqslant 2\left(n_{1}+1\right)+r-v \\
\max _{i \geqslant 3, r}\left(\alpha_{r}^{1}, \alpha_{r}^{2}, \alpha_{r}^{i} / 2\right) \leqslant n_{1}+1}} e^{-M_{j}} g_{\alpha, \beta}^{3}\left(H_{\alpha, \beta}^{1}+H_{\alpha, \beta}^{2}\right)\right\}\left(0, y_{j}\right) ;
\end{array} \\
& A_{j, b}^{s}=\int_{w_{0}(x, t)}^{w(x, t)}\left\{e^{-M_{j} \partial_{x}^{n_{1}+1} u_{2}}\right. \\
& \left.\cdot \sum_{\substack{\alpha^{i}, \beta^{i} \\
\sum_{i}\left(\left|\alpha^{i}\right|+\mid \beta^{i}\right) \leqslant 2\left(n_{1}+1\right)+r-v \\
\max _{i>3, r}\left(\alpha_{r}^{1}, \alpha_{r}^{2}, \alpha_{r}^{i} / 2\right) \leqslant n_{1}+1}} e^{-M_{j}} g_{\alpha, \beta}^{3}\left(H_{\alpha, \beta}^{1}+H_{\alpha, \beta}^{2}\right)\right\}\left(0, y_{j}\right) \\
& \left.\sum_{\substack{\alpha^{i}, \beta^{i} \\
\sum_{i}\left(\left|\alpha^{i}\right|+\left|\beta^{i}\right|\right) \leqslant 2\left(n_{1}+1\right)+r-v \\
\max _{i \geqslant 3, r}\left(\alpha_{r}^{1}, \alpha_{r}^{2}, \alpha_{r}^{i} / 2\right) \leqslant n_{1}+1}} e^{-M_{j}} g_{\alpha, \beta}^{3}\left(H_{\alpha, \beta}^{1}+H_{\alpha, \beta}^{2}\right)\right\}\left(0, y_{j}\right) \\
& \begin{array}{l}
\left.\sum_{\substack{\alpha^{i}, \beta^{i} \\
\sum_{i}\left(\left|\alpha^{i}\right|+\left|\beta^{i}\right|\right) \leqslant 2\left(n_{1}+1\right)+r-v \\
\max _{i \geqslant 3, r}\left(\alpha_{r}^{1}, \alpha_{r}^{2}, \alpha_{r}^{i} / 2\right) \leqslant n_{1}+1}} e^{-M_{j}} g_{\alpha, \beta}^{3}\left(H_{\alpha, \beta}^{1}+H_{\alpha, \beta}^{2}\right)\right\}\left(0, y_{j}\right) ;
\end{array}
\end{aligned}
$$

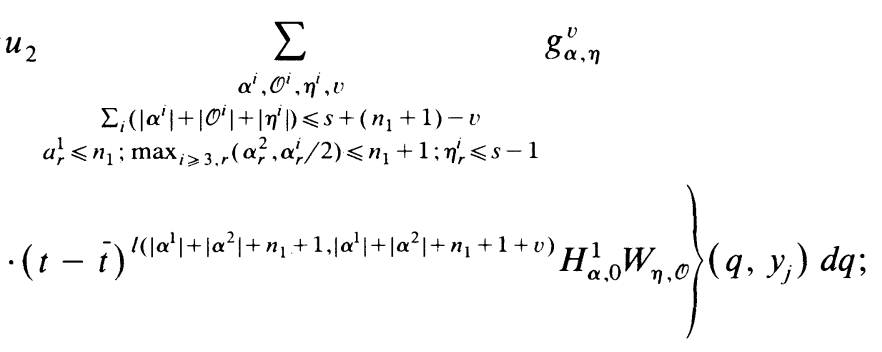

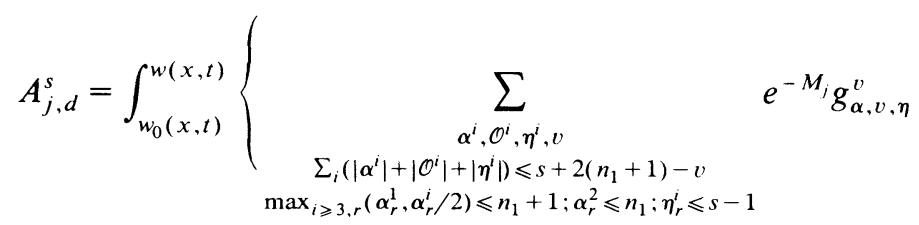

$$
\begin{aligned}
& \left.\cdot(t-\bar{t})^{l\left(\left|\alpha^{1}\right|+\left|\alpha^{2}\right|,\left|\alpha^{1}\right|+\left|\alpha^{2}\right|+v\right)} H_{\alpha, 0} W_{\eta, \mathcal{O}}\right\}\left(q, y_{j}\right) d q \\
& A_{j, p}^{s}=\sum_{\alpha^{i}, \beta^{i}, \eta^{i}, \mathcal{O}^{i}, v} \\
& \sum_{i}\left(\left|\alpha^{i}\right|+\left|\beta^{i}\right|+\left|\eta^{i}\right|+\left|O^{i}\right|\right) \leqslant s-1+2\left(n_{1}+1\right)-v \\
& \max _{i \geqslant 3, r}\left(\alpha_{r}^{1}, \alpha_{r}^{i} / 2\right) \leqslant n_{1}+1 ; \alpha_{r}^{2} \leqslant n_{1} \\
& \eta_{r}^{i} \leqslant s-1, \mathcal{O}_{r}^{i} \leqslant s-1 \\
& \cdot\left\{g_{\alpha, \beta, \eta, \mathcal{O}}^{v}(t-\bar{t})^{l\left(\left|\alpha^{1}\right|+\left|\alpha^{2}\right| .\left|\alpha^{1}\right|+\left|\alpha^{2}\right|+v\right)+p-1} H_{\alpha, \beta}^{1} W_{\eta, \mathcal{O}}\right\}(x, t)
\end{aligned}
$$


where the functions $g^{v}$ depend smoothly on $\mathbf{u}, w(x, t), y_{j}(x, t), x\left(q, y_{j}(c, t)\right)$, and $t\left(q, v_{j}(x, t)\right)$.

To understand how these complicated expressions arise, we refer to (4.25). Each of the $A_{j}^{s}$ 's is the result of the iterated application of rules (4.24), being careful to replace $X_{1}^{s}\left(\partial_{t} X_{1}\right)^{n_{1}+1} u_{i}, i \geqslant 3$, each time by their expression in terms of $A_{i}^{s}$ and $B_{i}^{s}$. We want to show that for $k \leqslant 2\left(n_{1}+1\right)(p-1)+p$ the integrals involved make sense and (4.28) actually defines a function that is continuous across $C_{j} \backslash\{(0, t)\}$. The basic observation is that for $s \leqslant 2\left(n_{1}+1\right)(p-1)$ the quantities

$$
K_{s}^{i}=(t-\bar{t})^{2\left(n_{1}+1\right)(p-1)-s} X_{i}^{2\left(n_{1}+1\right)(p-1)+p-s}\left(\partial_{x}^{n_{1}+1} u_{1} \partial_{x}^{n_{1}+1} u_{2}\right), \quad i=1,2,
$$

are integrable along certain segments of $C_{j}$, namely on the right of the curve $w=0$ if $i=1$, and on the left if $i=2$. In fact, using the inductive hypothesis, we have, in the region $w>0$ where $x>c_{1}(t-\bar{t})^{p}$,

$$
\left|K_{s}^{1}\right| \leqslant c_{2}(t-\bar{t})^{2\left(n_{1}+1\right)(p-1)-s} x^{-\left(2\left(n_{1}+1\right)(p-1)+p-s\right) / p+1 / p} \leqslant c_{3} x^{-1+1 / p} .
$$

In the region $w<0$, where $y(x, t) \geqslant c_{4}(t-\bar{t})^{p}$, we have, similarly,

$$
\left|K_{s}^{2}\right| \leqslant c_{5}(y(x, t))^{-1+1 / p} \text {. }
$$

Lemma 2.2 insures that the functions $x^{-1+1 / p}$ and $y^{-1+1 / p}$ are integrable along any $j$ th characteristic, $C_{j}$ included. Hence, the integrals in $A_{k, a}^{k}$ and $B_{j, a}^{k}$ make sense and are continuous across $C_{j}$ for $k \leqslant 2\left(n_{1}+1\right)(p-1)+p$ (corresponding to a derivative of order less than or equal to $\left.\left(n_{1}+n_{1}+1+2\right) p\right)$, being a linear combination of terms, the most singular of which are similar to $K_{k}^{1}$ and $K_{k}^{2}$ for

$$
k \leqslant 2\left(n_{1}+1\right)(p-1)+p .
$$

The terms at $\left(0, y_{j}\right)$ in $A_{j, a}^{k}$ and $B_{j, a}^{k}$ tend to zero as $(x, t)$ tends to $C_{j}$. In fact, recalling Remark 4.2, at $\left(0, y_{j}\right)$ estimates (4.6)(i), (ii) both apply. Hence, using the inductive hypothesis it is easy to recognize that the most singular term can be bounded as follows:

$$
\begin{aligned}
& \mid X_{1}^{k-1-r}\left\{(t-\bar{t})^{2\left(n_{1}+1\right)(p-1)+p-1-v}\left(X_{1}^{r-v}+\partial_{t}^{r-v}\right)\right. \\
& \left.\cdot\left(\partial_{x}^{n_{1}+1} u_{1} \partial_{x}^{n_{1}+1} u_{2}\right)\right\}\left(x\left(0, y_{j}\right), t\left(0, y_{j}\right)\right) \mid \\
& \leqslant c_{1} \sum_{s=0}^{k-1-r} \mid\left\{(t-\bar{t})^{2\left(n_{1}+1\right)(p-1)+p-1-v-s}\left(X_{1}+\partial_{t}\right)^{k-1-r-s}\right. \\
& \left.\cdot\left(X_{1}^{r-v}+\partial_{t}^{r-v}\right)\left(\partial_{x}^{n_{1}+1} u_{1} \partial_{x}^{n_{1}+1} u_{2}\right)\right\}\left(0, y_{j}\right) \mid .
\end{aligned}
$$

Now if $(x, t)$ tends to $C_{j}$ then $y_{j} \rightarrow \bar{t}$ and so does $t\left(0, y_{j}(x, t)\right)$. Hence, the left side of (4.36) goes to zero for $k \leqslant 2\left(n_{1}+1\right)(p-1)+p$ unless $r=v=0, s=k-1$, $k=2\left(n_{1}+1\right)(p-1)+p$. In that case one has to prove the continuity of $\left(\partial_{x}^{n_{1}+1} u_{1} \partial_{x}^{n_{1}+1} u_{2}\right)\left(0, y_{j}\right)$ across $(0, \bar{t})$. Here the parity of $p$ plays a role. In fact, since $p$ is even, the two characteristics $C_{1}$ and $C_{2}$ do not cross, and $\left(\partial_{x}^{n_{1}+1} u_{1} \partial_{x}^{n_{1}+1} u_{2}\right)\left(0, y_{j}\right)$ is continuous across $(0, \bar{t})$ even if $n_{1}=n_{2}$, so both derivatives have jumps. (If $p$ is odd this is not true, and this is the only difference in the two cases.) This ends the proof that $A_{j, a}^{k}$ and $B_{j, a}^{k}$ are well defined and continuous across $C_{j} \backslash\{(0, \bar{t})\}$. The same is true for $A_{j, b}^{k}, A_{j, d}^{k}, B_{j, b}^{k}, B_{j, d}^{k}$ since the functions present in those integrals are bounded and continuous by the inductive hypothesis and their recursive definition. 
Finally, $A_{j, p}^{k}$ and $B_{j, p}^{k}$ are continuous across $C_{j}$, since in their expression only derivatives of order at most $2\left(n_{1}+1\right)+k-1$ appear, and these are continuous by the inductive hypothesis.

The proof by induction of (4.32) and (4.33) is a long but straightforward application of the rules in (4.24). (See [7] for details.)

Step 4. Proofs of the bounds on $X_{i}^{k+1-s} \partial_{x}^{s} u_{j}$ and $X_{i}^{k-2\left(n_{1}+1\right)} L^{2\left(n_{1}+1\right)} u_{k}, i=1,2$, $j \geqslant 3$.

In the proof of the continuity of $X_{1}^{k+1-2\left(n_{1}+1\right)} L^{2\left(n_{1}+1\right)} u_{j}$ contained in Step 3, we used the inductive hypothesis at Step $k$, but we have been very careful not to create any derivative of order $k+1$ (except, of course, of $\mathbf{u}^{0}$ ). We can then use formula (4.28), with $k$ replaced by $k+1$, to prove (4.6)(iii). We focus on $X_{1}^{k-2\left(n_{1}+1\right)} L^{2\left(n_{1}+1\right)} u_{j}$; $\partial_{t}^{k-2\left(n_{1}+1\right)} L^{2\left(n_{1}+1\right)} u_{j}$ can be treated likewise.

We first expand $X_{1}^{k-l+1} e^{M_{j}}$ using (4.30). Examining the right side of (4.28) we easily recognize that the only unbounded functions present are $A_{j, p}^{l_{1}}$ and $B_{j, p}^{l_{2}}$, with $0 \leqslant l_{1}, l_{2} \leqslant k+1$. Recalling definitions (4.32) and (4.33) we have, for $l_{1}+l_{2} \leqslant k+$ $1-2\left(n_{1}+1\right)$,

$$
\begin{gathered}
\left|A_{j, p}^{l_{1}(x, t)} B_{j, p}^{l_{1}}(x, t)\right| \\
\leqslant c_{1}\left\{\sum_{v_{1}=0}^{l_{1}} \sum_{v_{2}=0}^{l_{2}}\left[(t-\bar{t})^{l\left(\left(n_{1}+1\right),\left(n_{1}+1\right)+v_{1}\right)+p-1} x^{-\left(l_{1}-v_{1}-1\right)}+\delta_{l_{1}, 0}\right]\right. \\
\left.\cdot\left[(t-\bar{t})^{l\left(n_{1}+1, n_{1}+1+v_{2}\right)+p-1} x^{-\left(l_{2}-v_{2}-1\right) / p}+\delta_{l_{2}, 0}\right]\right\} \\
\leqslant c_{2}|x|^{-\left(k+1-2\left(n_{1}+1\right)-1\right) / p+\left(n_{1}+2\right)(1-1 / p)} .
\end{gathered}
$$

The bounds on $X_{1}^{k-s} \partial_{x}^{s} u_{j}$ now follow easily from (4.6)(iii). This ends the proof of the inductive hypothesis (4.6).

We have then proved the following theorem:

THEOREM 4.1. Let $\mathbf{u}$ be the solution of system (2.1) with initial data satisfying condition (2.3). Then

$$
u \in C^{\left(n_{1}+n_{1}+1+2\right) p}\left(R_{T} \backslash\left(C_{1} \cup C_{2}\right)\right) .
$$

5. A counterexample. In this section we present an example that shows how the regularity result across $C_{j}$ obtained in $\$ 4$ cannot be improved in general. We use the same example presented in [4] but with different initial data. We will constantly refer to the quoted paper for further details. Consider the following system:

$$
\begin{aligned}
\left(\partial / \partial t+p(t-1)^{p-1} \partial / \partial_{x}\right) v=0, & v(x, 0)=v^{0}(x), \\
\partial / \partial_{t} w=v, & w(x, 0)=0, \\
\left(\partial / \partial_{t}+\partial / \partial_{x}\right) z=D_{+} z=w v, & z(x, 0)=0,
\end{aligned}
$$

where

$$
v^{0}(x)=\left\{\begin{aligned}
(x-1)^{n_{1}+1}, & x \leqslant 1 \\
2(x-1)^{n_{1}+1}, & x \geqslant 1
\end{aligned}\right.
$$

Let $\Omega_{1}$ be the region shadowed in Figure 5.1 and $\chi_{\Omega_{1}}$ its characteristic function. 


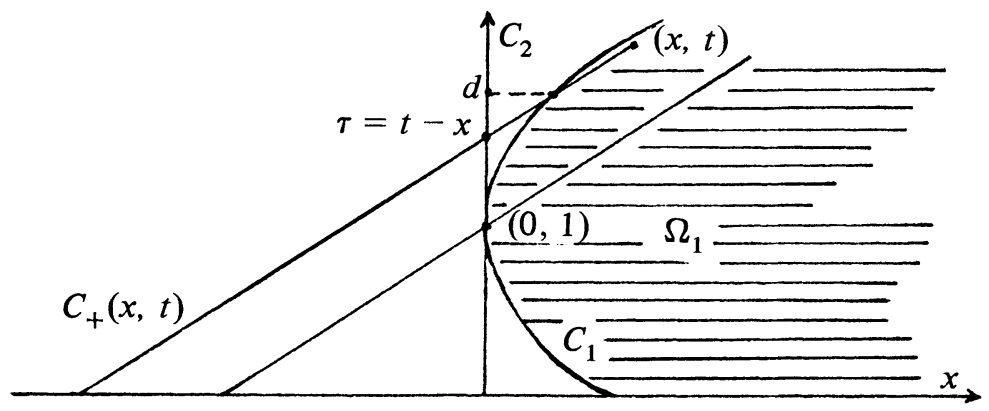

Figure 5.1

We have the following expressions for $v, w$, and $z$ :

$$
\begin{gathered}
v(x, t)=v^{0}\left(x-(t-1)^{p}+1\right)= \begin{cases}\left(x-(t-1)^{p}\right)^{n_{1}+1}=\bar{v}(x, t) & \text { in } R^{2} \backslash \Omega_{1}, \\
2\left(x-(t-1)^{p}\right)^{n_{1}+1}=2 \bar{v}(x, t) & \text { in } \Omega_{1},\end{cases} \\
w(x, t)=\int_{0}^{t} \bar{v}(x, q)\left(1+\chi_{\Omega_{1}}\right) d q=A(x, t)+B(x, t),
\end{gathered}
$$

where

$$
A(x, t)=\int_{0}^{t} \bar{v}(x, q) \chi_{\Omega_{1}} d z
$$

$$
z(x, t)=\int_{C_{+}(x, t)}(A+B) \bar{v}\left(1+\chi_{\Omega_{1}}\right)(x(s), t(s)) d s,
$$

where $x(s), t(s)$ is the arc length parametrization of the backward $D_{+}$characteristic $C_{+}(x, t)$ from $(x, t)$. It is easy to realize that

$$
\int_{C_{+}(x, t)}\left[B \bar{v}\left(1+\chi_{\Omega_{1}}\right)+2 A \bar{v}\right] \text { is smooth in } \Omega_{1} \backslash\left(C_{4} \cup C_{5}\right) .
$$

Moreover,

(5.3) $z(x, t)=\int_{C_{+}(x, t)}\left[B \bar{v}\left(1+\chi_{\Omega_{1}}\right)+2 A \bar{v}\right] d s-\int_{C_{+}(x, t)}\left[A \bar{v} \chi_{R^{2} \backslash \Omega_{1}}\right] d s$.

Clearly, $A$ is different from zero only inside or above the region $\Omega_{1}$. The function inside the last integral in (5.3) is then always zero if $(x, t)$ is below $C_{3}$. If $(x, t)$ is above $C_{3}$ the only part of the path of integration on which $A x_{R^{2} \backslash \Omega_{1}}$ does not vanish is the segment with endpoints $(0, \tau)$ and $\left((d-1)^{p}, d\right)$ (see Figure 5.1). In that region

$$
A(x, t)=\int_{1-x^{1 / p}}^{1+x^{1 / p}}\left(x-(q-1)^{p}\right)^{n_{1}+1} d q=c_{1} x^{n_{1}+1+1 / p} .
$$


Then

$$
\begin{aligned}
& \int_{0}^{(d-1)^{p}}(A \bar{v})(q, q+\tau) d q \\
& \quad=c_{1} \int_{0}^{(d-1)^{p}} q^{n_{1}+1+1 / p}\left(q-(q+\tau-1)^{p}\right)^{n_{1}+1} d q \\
& \quad=(d-1)^{\left(n_{1}+n_{1}+1+2\right) p+1} c_{1} \int_{0}^{1} r^{n_{1}+1+1 / p}\left(r-\left[r(d-1)^{p-1}+\frac{\tau-1}{d-1}\right]^{p}\right)^{n_{1}+1} d r \\
& \quad=(d-1)^{\left(n_{1}+n_{1}+1+2\right) p+1} c_{1} \int_{0}^{1} r^{n_{1}+1+1 / p}\left(r-\left[(r-1)(d-1)^{p-1}+1\right]^{p}\right)^{n_{1}+1} d r .
\end{aligned}
$$

where we used that $\tau-1=(\tau-d)+(d-1)=-(d-1)^{p}+d-1$. The r.h.s. of (5.3) is $\left(n_{1}+n_{1}+1+2\right) p$-times differentiable across $C_{3}$, but has a jump in the $\left(n_{1}+n_{1}+1+1\right) p+1$ derivative, since the last integral does not vanish.

ACKNOWLEDGEMENTS. I would like to thank Professor M. Reed for many valuable discussions and helpful suggestions.

\section{REFERENCES}

1. J. Rauch and M. Reed, Propagation of singularities for semilinear hyperbolic equations in one space variable, Ann. of Math. (2) 111 (1980), 531-552.

2. ___ Jump discontinuities of semilinear hyperbolic systems in two variables: creation and propagation, Comm. Math. Phys. 81 (1981), 203-227.

3. , Nonlinear microlocal analysis of semilinear hyperbolic systems in one space dimension, Duke Math. J. 49 (1982), 397-475.

4. Propagation of singularities in nonstrictly hyperbolic systems: examples, Comm. Pure Appl. Math. 35 (1982), 555-565.

5. J. Ralston, On propagation of singularities in solutions of symmetric hyperbolic partial differential operators, Comm. Partial Differential Equations 1 (1976), 87-133.

6. K. Taniguchi and Y. Tozaki, A hyperbolic equation with double characteristic which has a solution with branching singularities, Math. Japon. 22 (1980), 279-300.

7. L. Micheli, Ph. D. Thesis, Duke Univ., 1984.

Department of Mathematics, Duke University, Durham, North Carolina 27706

Current address: Dipartimento di Matematica, Università di Roma “La Sapienza”, P. A. Moro, 00185 Roma, Italy 